\title{
Smoothing it Out: Empirical and Simulation Results for Disentangled Realized Covariances*
}

\author{
Harry Vander Elst ${ }^{a}$ \\ David Veredas ${ }^{a, b}$ \\ ${ }^{a}$ ECARES, Solvay Brussels School of Economics and Management, Université libre de Bruxelles \\ ${ }^{b}$ Institute of Information Theory and Automation, Academy of Sciences of the Czech Republic
}

\begin{abstract}
We study the class of disentangled realized estimators for the integrated covariance matrix of Brownian semimartingales with finite activity jumps. These estimators separate correlations and volatilities. We analyze different combinations of quantile- and median-based realized volatilities, and four estimators of realized correlations with three synchronization schemes. Their finite sample properties are studied under four data generating processes, in presence, or not, of microstructure noise, and under synchronous and asynchronous trading. The main finding is that the pre-averaged version of disentangled estimators based on Gaussian ranks (for the correlations) and median deviations (for the volatilities) provide a precise, computationally efficient, and easy alternative to measure integrated covariances on basis of noisy and asynchronous prices. A minimum variance portfolio application shows the superiority of this disentangled realized estimator in terms of numerous performance metrics.
\end{abstract}

Keywords: Realized measures, noise, jumps, synchronization.

JEL Classification:C58

*Acknowledgments. This work was partly done while Harry Vander Elst visited CREATES at Aarhus University and when David Veredas held the Bank of Santander Chair of Excellence at the Statistics Department of the University Carlos III de Madrid. Harry Vander Elst gratefully acknowledges financial support from a FRESH grant and David Veredas from the the Czech Science Foundation under project No. 13-32263S. We are also grateful to Asger Lunde, Kris Boudt, Torben Andersen, and the conference participants at CFE13 (London, December 2013) and the SoFiE annual conference 20155, as well as the seminars participants at CREATES for insightful remarks. A previous draft of the paper has circulated under the title "Disentangled Jump-Robust Realized Covariances and Correlations with Non-Synchronous Prices". Any error and inaccuracy are ours. 


\section{Introduction}

Many activities in financial institutions - such as risk management, portfolio selection, and asset pricing require precise measures that summarize the relationships between risk factors. Among these measures, correlations and volatilities are of paramount importance as they provide, on the one hand, estimates of the links between assets and, on the other hand, the inputs for various risk measures.

Realized correlations and volatilities are nonparametric estimators of the ex-post variation of prices. In both the univariate and multivariate cases, the baseline estimators (simply obtained by summing intraday squared returns or intraday product of returns) face numerous drawbacks.

First, empirical properties of asset prices suggest the existence of jumps. Jumps enable to accommodate fat tails in the empirical density of returns and smiles in volatility surfaces of option prices. Models for jumps can be of two types: either with finite amount of large jumps or with infinite amount of small jumps. Jumps introduce an additional source of variation in prices which is of interest for many purposes, but may may lead to biases in covariance measurements. Several estimators are able to separate these sources of variations. For the univariate case see e.g. bipower variation (Barndorff-Nielsen \& Shephard (2004b)), quantile-based realized variances (Christensen et al. (2010b)), and MinRV and MedRV (Andersen et al. (2012)). In the multivariate case, bipower covariations are proposed in Barndorff-Nielsen \& Shephard (2004a), thresholds covariances in Mancini \& Gobbi (2012), outlyingness weighted covariances in Boudt et al. (2011b), and disentangled covariances in Boudt et al. (2012).

Second, intraday prices are unreliably recorded, as they do not necessarily correspond to those at which the underlying asset has been traded, as pointed out in Zhou (1996). This phenomenon, labeled as market microstructure noise (noise henceforth), affects significantly the properties of realized measures. Several solutions are provided in the literature both for realized variances and covariances. They include, among others, sparse sampling (Andersen et al. (2001) and Bandi \& Russell (2008)), multi-scale estimators (Zhang et al. (2005) and Zhang (2006)), pre-averaging techniques (Podolskij \& Vetter (2009), Jacod et al. (2009), Christensen et al. (2010a) and Christensen et al. (2013)), realized kernels (Barndorff-Nielsen et al. (2008) and Barndorff-Nielsen et al. (2011)), pseudo-maximum likelihood techniques (Aït-Sahalia et al. (2010)), and measures based on the Kalman filter and the EM algorithm (Shephard \& Xiu (2012) and Corsi et al. (2014)).

Third, and this is specific to the multivariate setup, while price series are non-synchronous and discrete, the underlying theory of realized estimators is based on continuous stochastic processes. As a result, most of the multivariate tools require synchronous data. Several estimators and sampling methods have been proposed to cope with non-synchronous transactions. A first generation of interpolation techniques are documented in Dacorogna et al. (2001) and have been extensively used. Despite their success, they are known to produce downward biases in correlations at very high frequencies. The latter phenomenon, known as the Epps effect, is well documented in the empirical literature (see Epps (1979) and Reno (2003)). Modern synchronization devices have been proposed recently to construct 
homogeneous prices (see e.g. Hayashi \& Yoshida (2005), Barndorff-Nielsen et al. (2011), Corsi \& Audrino (2012) and Aït-Sahalia et al. (2010)).

This article investigates the properties of the class of disentangled realized covariance estimators (DRC) introduced by Boudt et al. (2012), i.e. realized covariances computed as the product of realized volatilities and correlations. The reason for disentangling covariances into correlations and volatilities is the optimal use of the available information. Measuring separately volatilities and correlations allows to measure each component using the largest amount of available information. Indeed, as returns only need to be synchronized for correlations, volatilities are measured using the full sample of data. This approach has potential advantages in terms of precision for the estimation. In fact, separating the estimation of correlations and volatilities is not an uncommon practice in econometrics. In the parametric multivariate GARCH set up, Bollerslev (1990), Tse \& Tsui (2000), and Engle (2002) propose equivalent approaches with CCC and DCC models (see Bauwens et al. (2006) for an extensive review). Halbleib \& Voev (2011) propose a mixed approach, combining the DCC for the correlations and the realized estimators for the volatilities.

Our main contribution is twofold. First, we investigate the best estimator among the class of DRC. Namely we look for the estimator that combines robustness to jumps and resilience to noise and asynchronicity. Second, we show that in finite samples the chosen estimator compares well with a wide array of competitors, and under challenging data generating processes. The Monte Carlo study is based on four different models that are frequently used in the literature. For testing our estimators in a realistic setting, these models are simulated along with different components to accommodate finite activity jumps, microstructure noise, and asynchronous trading. We find that the pre-averaged version of DRC computed with realized Gaussian ranks for the correlations and median-based realized volatilities provide the most precise results in presence of jumps. Moreover, this estimator is simple to program, computationally fast, and the estimated matrix is positive definite.

We empirically assess the goodness of disentangled realized covariances through an indirect evaluation based on a minimum variance portfolio management exercise. Data represent the largest companies traded on the NYSE. Competing estimators are also evaluated in the application. We find that disentangled realized covariances can be used reliably with forecasting models such as the HEAVY of Noureldin et al. (2012). Finally, we underline different empirical implications from the use of different forecasting models.

Throughout the paper, we use the following notations, unless explicitly stated otherwise: i) $p$ denotes the dimension of the random vector of returns, which has a covariance matrix with elements generically indexed by $i$ and $j$, ii) $t$ denotes time (measured in low frequency, typically a day), iii) $N$ is the number of high frequency observations (intraday observations if $t$ is measured in days) with index $m$ (i.e. $m=$ $0, \ldots, N)$, iv) every day is divided in $K$ blocks or subsamples indexed by $q$ (i.e. $q=1, \ldots, K$ ), and $k$ is the number of observations in each block or subsample. The hierarchy of frequency is therefore: 1 day composed by $N$ intraday observations, divided in $K$ blocks (or subsamples) with $k$ observations within 
each block (or subsample). Characters in bold denote vectors and matrices.

The rest of the paper is organized as follows. In Section 2 we first introduce the data generating process, notation, and the class of disentangled estimators. In Section 3, we review several procedures to handle microstructure noise and in Section 4 we define several synchronization schemes. We report the results of the extensive Monte Carlo study in section 5. Section 6 presents the gains of the disentangled estimators in terms of returns on investment. Section 7 concludes. Additional results are reported in the Appendix.

\section{Jump-robust covariation measurement}

We consider a $p$-dimensional random vector of no-arbitrage log-prices denoted $\left\{\mathbf{X}_{t}\right\}_{t \geq 0}$ and defined on a filtered probability space $\left(\Omega, \mathcal{A},\left(\mathcal{A}_{t}\right)_{t \in[0,1]}, \mathcal{P}\right)$. We assume that the process is adapted to the filtration $\left(\mathcal{A}_{t}\right)_{t \in[0,1]}$ and that the vector of log-prices behaves as an Itō semimartingale with finite activity jumps:

$$
\mathbf{X}_{t}=\int_{0}^{t} \boldsymbol{\mu}_{u} d \mathbf{u}+\int_{0}^{t} \boldsymbol{\Lambda}_{u} d \mathbf{W}_{u}+\sum_{0 \leq s \leq t} \mathbf{J}_{s} .
$$

The process $\boldsymbol{\mu}_{u}$ is locally element-wise bounded predictable, and the elements of $\boldsymbol{\Lambda}_{u}$ are adapted cádlag processes such that $\boldsymbol{\Sigma}_{u}=\boldsymbol{\Lambda}_{u}^{T} \boldsymbol{\Lambda}_{u}$. The matrix $\boldsymbol{\Sigma}_{u}$ denotes the spot (or instantaneous) covariance matrix of the process. The random vector $\mathbf{W}_{u}$ denotes a $p$-dimensional standard Brownian motion and $\mathrm{J}_{s}$ denotes the jumps magnitude. Jumps are driven by a finite activity counting process $\boldsymbol{N}_{\boldsymbol{t}}$ such that $E\left[\boldsymbol{N}_{\boldsymbol{t}}\right]<\infty$. The component $\int_{0}^{t} \boldsymbol{\mu}_{u} d \mathbf{u}+\int_{0}^{t} \boldsymbol{\Lambda}_{u} d \mathbf{W}_{u}$ in (1) is the continuous part and denoted by $\mathbf{X}_{t}^{c}$, so that

$$
\mathbf{X}_{t}=\mathbf{X}_{t}^{c}+\sum_{0 \leq s \leq t} \mathbf{J}_{s}
$$

Since we are interested in robustness to large unexpected deviations in asset prices, we limit to finite activity jumps driven by a Poisson process as described in (1), despite the fact that infinite activity jumps processes are also considered in practice (e.g. Carr et al. (2002)). Further work might integrate finite and infinite activity jumps, and test whether it is possible to separate between large and small jumps in the price process using the statistics presented hereafter.

The period of interest is [0,1] (e.g. one day). We denote by $\pi_{N}$ an ordered set of times such that $0=t_{0}<t_{1}<\ldots<t_{m}<\ldots<t_{N}=1$ forming a partition (see definition 1 below) of the period considered, and $\mu\left(\pi_{N}\right)$ is the mesh of the partition. In this setup, the $\pi_{N}$-quadratic variation process of $\left\{\mathbf{X}_{t}\right\}_{t \geq 0}$ coincide with the baseline realized covariance and is defined as the random process

$$
\mathbf{Q}_{\pi_{N}}\left(\mathbf{X}_{t}\right)=\sum_{m=1}^{N}\left(\mathbf{X}_{t_{m} \wedge t}-\mathbf{X}_{t_{m-1} \wedge t}\right)\left(\mathbf{X}_{t_{m} \wedge t}-\mathbf{X}_{t_{m-1} \wedge t}\right)^{T} .
$$

If $\mathbf{Q}_{\pi_{N}}\left(\mathbf{X}_{t}\right)$ converges in probability to a process $\left\{\mathbf{V}_{t}\right\}_{t \in[0,1]}$ for any sequence partition $\pi_{N}$ over the interval [0,1] such that $\boldsymbol{\mu}\left(\pi_{N}\right) \rightarrow 0$ as $N \rightarrow \infty$, then we call $\left\{\mathbf{V}_{t}\right\}_{t \in[0,1]}$ the quadratic variation, which for simplicity we denote by $[\mathbf{X}]_{t}$. Assuming $\mathbf{X}_{0}=0$, it is well known for Itō semimartingales that

$$
[\mathbf{X}]_{t}=[\mathbf{X}]_{t}^{c}+\sum_{0 \leq s \leq t} \Delta \mathbf{J}_{s} \Delta \mathbf{J}_{s}^{T}
$$


where $[\mathbf{X}]_{t}^{c}=\int_{0}^{t} \Sigma_{u} d \mathbf{u}$ is the "path-by-path" continuous part of the quadratic variation (Protter (2004)), which also corresponds to the quadratic variation of the continuous part $\mathbf{X}_{t}^{c}$. The realized covariance $\mathbf{Q}_{\pi_{N}}\left(\mathbf{X}_{t}\right)$ can therefore be seen as a finite sample measure of risk composed of two sources: risks related to the Brownian component and risks related to the finite activity jumps.

Considering $\sigma_{u}^{i, j}$ as the $i$-th row and $j$-th column element of $\Sigma_{u}$, we have that $\sigma_{u}^{i, j}=\rho_{u}^{i, j} \sigma_{u}^{i} \sigma_{u}^{j}$, where $\sigma_{u}^{i}$ and $\sigma_{u}^{j}$ are the spot volatilities and $\rho_{u}^{i, j}$ is the spot correlation. Our object of interest is the estimation of $[\mathbf{X}]_{1}^{c}$ by estimating separately the elements $\rho_{u}^{i, j}, \sigma_{u}^{i}$ and $\sigma_{u}^{j}$

$$
[X]_{1}^{c, i, j}=\int_{0}^{1} \rho_{u}^{i, j} \sigma_{u}^{i} \sigma_{u}^{j} d u
$$

In other words, we are interested in a jump robust estimator of the daily integrated covariation by considering separately spot correlations and volatilities. As mentioned above, this approach allows to use the full data sample for volatilities $\sigma_{u}^{i} i=1, \ldots, p$ as no synchronization technique is required for their estimation.

We now define more rigorously the partitions one might encounter: ${ }^{1}$

Definition 1 Let $\pi_{N_{i}}:=\left[0=t_{0}^{i}<t_{1}^{i}<\ldots<t_{m}^{i}<\ldots<t_{N_{i}-1}^{i}<t_{N_{i}}^{i}=1\right]$ be the partition on the time interval $[0,1]$ for asset $i$. Likewise for asset $j$. We say that $\pi_{N_{i}}$ and $\pi_{N_{j}}$ are

1. synchronous and evenly spaced: $N_{i}=N_{j}, \pi_{N_{i}}=\pi_{N_{j}}$ and $t_{m}^{i}-t_{m-1}^{i}=t_{m}^{j}-t_{m-1}^{j}=\frac{1}{N}$.

2. synchronous and irregularly spaced: $N_{i}=N_{j}, \pi_{N_{i}}=\pi_{N_{j}}$ but time intervals between prices are not deterministic.

3. partially asynchronous: $\pi_{N_{i}} \neq \pi_{N_{j}}$ but we might have that $\pi_{N_{i}} \cap \pi_{N_{j}} \neq\{0,1\}$, i.e. there may be common points in the two sets except the first and the last (both sets are partitions of the same interval).

4. completely asynchronous: $\pi_{N_{i}} \neq \pi_{N_{j}}$ and $\pi_{N_{i}} \cap \pi_{N_{j}}=\{0,1\}$.

Figure 1 provides a diagrammatic representation of the partitions. The upper two lines corresponds to a partition of type 1 . The arrival times are regularly spaced and synchronized. The next two lines represent the partition of type 2 , in which arrival times are also synchronized but irregularly spaced. The bottom half of the figure represents the partitions 3 and 4 in which observations are asynchronized, though in partition 3 there maybe sporadic common arrival times, an event excluded in partition 4 .

In order to introduce the class of estimators in a clear way, we first assume that $\pi_{N_{i}}$ and $\pi_{N_{j}}$ are of type 1 . Then, the time intervals are denoted as $\Delta_{m}^{N} t=t_{m}-t_{m-1}$ and equal to $1 / N$. Let $\Delta_{m}^{N} \mathbf{X}=$ $\mathbf{X}_{m / N}-\mathbf{X}_{(m-1) / N}=\left(X_{i, m / N}-X_{i,(m-1) / N}, X_{j, m / N}-X_{j,(m-1) / N}\right)$ be the vector of synchronous log-returns computed over the period of interest. ${ }^{2}$ The returns scaled (by time) are $\Delta_{m}^{N} \mathbf{X}^{*}=\left(\Delta_{m}^{N} t\right)^{-\frac{1}{2}} \Delta_{m}^{N} \mathbf{X}=\sqrt{N} \Delta_{m}^{N} \mathbf{X}$.

We construct $K$ non-overlapping smaller subsets (or blocks) of returns containing each $k$ data points,

\footnotetext{
${ }^{1}$ This definition is in terms of 2 assets and it can be generalized to any dimension.

${ }^{2}$ Note that since partitions are of type $1, \mathbf{X}_{t_{m}}=\mathbf{X}_{m / N}$.
} 
Asset 1

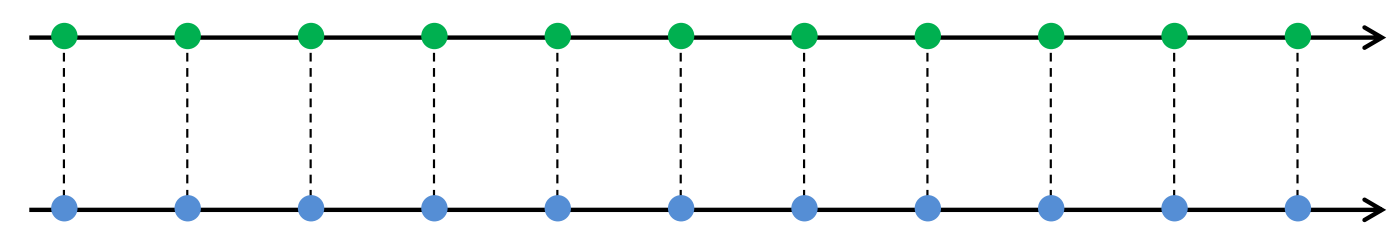

Asset 2

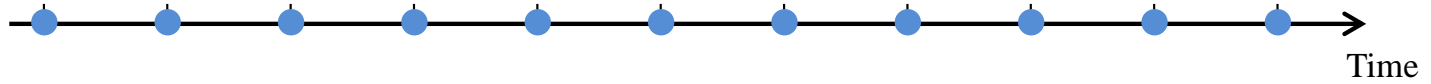

Asset 1

Asset 2

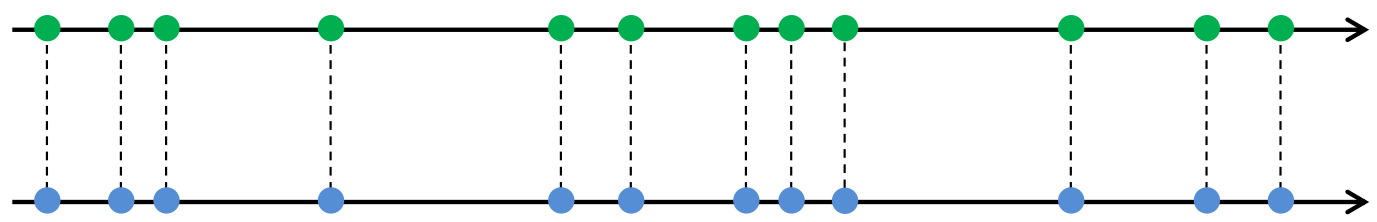

Time

Asset 1

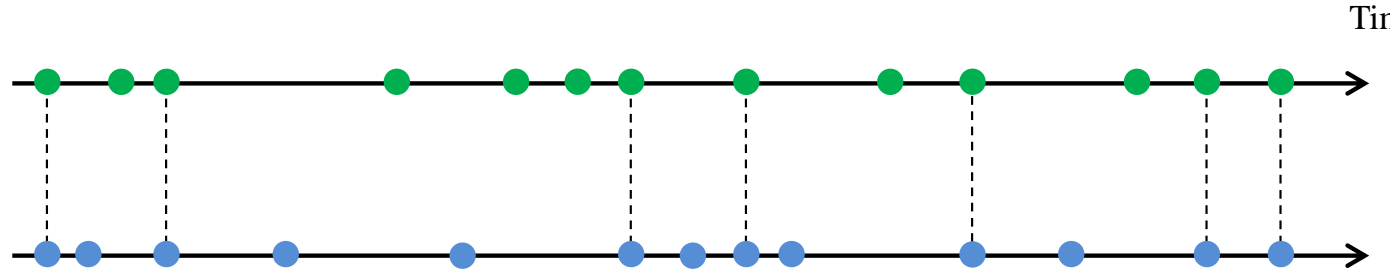

Asset 2

Time

Asset 1

Asset 2

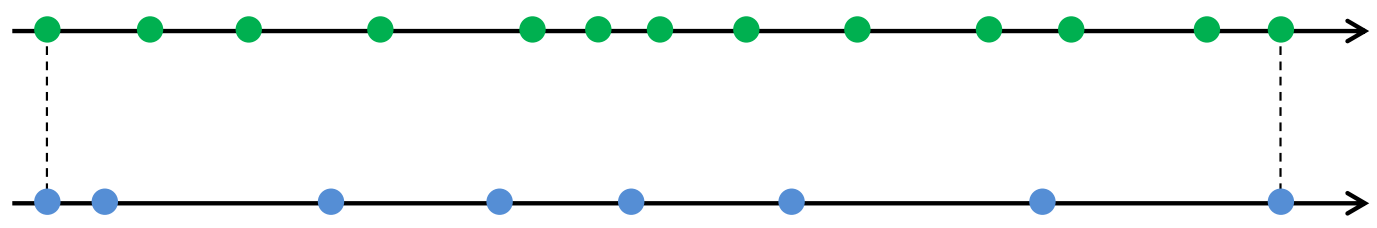

Time

Figure 1: This figure provides a diagrammatic representation of the four different partitions. The upper two lines correspond to a partition of type 1 . The arrival times are regularly spaced and synchronous. The next two lines represent the partition of type 2 , in which arrival times are also synchronous but irregularly spaced. The bottom half of the figure represents the partitions 3 and 4 in which observations are non-synchronous, though in partition 3 there maybe sporadic common arrival times, an event excluded in partition 4.

i.e. $N=K \times k .^{3}$ We denote by $B_{q}^{k} \mathbf{X}^{*}=\left(\Delta_{m}^{N} \mathbf{X}^{*}\right)_{(q-1) k+1 \leq m \leq q k}$ the subset of scaled returns contained in block $q$.

The class of Disentangled Realized Covariances between assets $i$ and $j$ is

$$
D R C^{i, j}(K)=\frac{1}{K} \sum_{q=1}^{K} r^{i, j}\left(B_{q}^{k} \mathbf{X}^{*}\right) v^{i}\left(B_{q}^{k} \mathbf{X}^{*}\right) v^{j}\left(B_{q}^{k} \mathbf{X}^{*}\right),
$$

where $r^{i, j}\left(B_{q}^{k} \mathbf{X}^{*}\right), v^{i}\left(B_{q}^{k} \mathbf{X}^{*}\right)$ and $v^{j}\left(B_{q}^{k} \mathbf{X}^{*}\right)$ denote respectively estimators of the correlation and volatilities for assets $i$ and $j$ computed using the scaled returns contained in block $q$. The choice of these estimators determine the properties of (5).

Indeed, many choices are available for $r^{i, j}\left(B_{q}^{k} \mathbf{X}^{*}\right), v^{i}\left(B_{q}^{k} \mathbf{X}^{*}\right)$ and $v^{j}\left(B_{q}^{k} \mathbf{X}^{*}\right)$. In this article we select some and analyze them. The methodology we use is somewhat inductive. We begin by studying the goodness of combinations of estimators for volatilities and correlations with Monte Carlo simulations.

\footnotetext{
${ }^{3}$ In some instances the last block may have a different amount of points depending on the initial amount of observations and the amount of blocks.
} 
Then, on basis of the finite sample results, we select the best estimator and compare its performances with benchmark jump-robust estimators from the literature. Our approach therefore extends Boudt et al. (2012) as several combinations of estimators are considered, different sampling schemes are used, and noise is introduced.

We close this sub-section with three remarks. The first concerns consistency and the jump-robust properties of the estimators. In a small interval, log-returns generated by the process (1) can be well approximated by a Brownian motion with constant covariance matrix. I.e. if $(q-1) k+1 \leq m \leq q k$, then

$\Delta_{m}^{N} \mathbf{X} \approx \Lambda_{\frac{(q-1) k+1}{N}} \Delta_{m}^{N} \mathbf{W}$ where the subindex $\frac{(q-1) k+1}{N}$ corresponds to the starting time of block $q$ (see e.g. Mykland \& Zhang (2009)). Since the approximated contiguous scaled returns located in block $q$ display constant spot covariance matrix and have distribution $N\left(0, \Sigma_{\frac{(q-1) k+1}{N}}\right)$, consistent robust estimators under Gaussianity enable to estimate the spot covariance matrix in each block. The average over blocks provides an estimator of the integrated covariance matrix that intuitively maps to a Riemann sum over time intervals.

Second, the finite activity jumps that we consider correspond to large unexpected movements. In a small block, these jumps can be mapped intuitively to outliers in the statistical sense. Estimators that are robust to outliers thus eliminate the effect of finite activity jumps.

Third, in the case of synchronous data $\left(\pi_{N_{i}}\right.$ and $\pi_{N_{j}}$ are of types 1 or 2 in definition 1$)$, measuring volatilities and correlations separately has of course little interest since no efficiency can be gained from disentangled estimation. Our class of estimator is best suited in the case of partitions $\pi_{N_{i}}$ and $\pi_{N_{j}}$ that are of type 3 or 4 , requiring a synchronization technique to estimate the spot correlations within each block. We discuss this point more in detail below.

\subsection{Estimation of the spot volatilities}

In this section we describe two jump-robust estimators of spot volatilities. They are derived from two classes of integrated volatility estimators proposed by Christensen et al. (2010b) and Andersen et al. (2012). We limit the analysis to these two classes as both are based on a blocking strategy, and hence dovetail into the class of disentangled realized covariances.

The estimator of integrated volatility proposed by Christensen et al. (2010b) is based on quantiles and defined, for asset $i$, as

$$
\operatorname{QRV}^{i}(\lambda, K)=\frac{1}{K} \sum_{q=1}^{K} \frac{s_{i}\left(B_{q}^{k} \mathbf{X}^{*}, \lambda\right)}{v(\lambda)},
$$

where $s_{i}\left(B_{q}^{k} \mathbf{X}^{*}, \lambda\right)=g_{\lambda k}^{2}\left(B_{q}^{k} \mathbf{X}^{*}\right)+g_{(1-\lambda) k+1}^{2}\left(B_{q}^{k} \mathbf{X}^{*}\right)$ and the function $g_{k}(\mathbf{x})=\mathbf{x}_{(k)}$ denotes the $k$-th order statistics. The parameter $\lambda$ is the probability level at which QRV is computed. ${ }^{4}$ The term $v(\lambda)$ in (6) is a scaling factor given by $v(\lambda)=\mathbb{E}\left[\left|U_{(\lambda m)}\right|^{2}+\left|U_{(m-\lambda m+1)}\right|^{2}\right]$, where $U_{(\lambda m)}$ is the $\lambda m$-th order statistics of a

\footnotetext{
${ }^{4}$ The information contained at more than one probability level can be exploited by considering $Q R V_{N}^{i}(\underline{\boldsymbol{\lambda}})=\boldsymbol{\alpha}^{T} \underline{\mathbf{Q R V}}{ }_{N}^{i}(\underline{\lambda})$, where $\boldsymbol{\alpha}$ is a vector (summing to one) that has the same size as $\underline{\lambda}$.
} 
sample of $m$ i.i.d. normal random variables $\left(U_{1}, \ldots, U_{m}\right)$. This scaling can be computed by simulation and ensures consistency of the estimator under Gaussianity.

The second class of estimators for integrated volatility is a generalization of those proposed by Andersen et al. (2012). For asset $i$ they are

$$
\begin{aligned}
\operatorname{MinRV}_{K}^{i} & =\frac{1}{\xi^{\operatorname{Min}}(k)} \sum_{q=1}^{K} \min \left(\left|B_{q}^{k} \mathbf{X}^{*}\right|\right)^{2} \text { and } \\
\operatorname{MedRV}_{K}^{i} & =\frac{1}{\xi^{\operatorname{Med}(k)}} \sum_{q=1}^{K} \operatorname{med}\left(\left|B_{q}^{k} \mathbf{X}^{*}\right|\right)^{2} .
\end{aligned}
$$

The scalings $\xi^{\operatorname{Min}}(k)$ and $\xi^{\text {Med }}(k)$ are such that the summands are consistent estimators of the spot volatility in the corresponding block under the assumption that observations are i.i.d. Gaussian. These constants are functions of the number of observations per block. Andersen et al. (2012) consider $k$ equal to 2 and 3 for $\operatorname{MinRV}_{N}^{i}$ and $M e d R V_{N}^{i}$ respectively, for which the scalings have closed-form expressions. For other values of $k$, they may have to be computed by simulations. ${ }^{5}$ If $k=1$, the scaling equals 1 for both estimators, which coincide with the baseline realized variance estimator. If $k \rightarrow \infty$, the scaling for MedRV converges to 2.198, while the scaling for MinRV increases exponentially to infinity. ${ }^{6}$ Because of this drawback, and because of the sensitivity to zero returns (leading to a bias towards zero), we do not consider the MinRV estimator. MedRV by contrast strikes a good balance between stability and jump robustness.

Based on $\operatorname{QRV}^{i}(\lambda, K)$ and $\operatorname{MedRV}_{K}^{i}$, the estimators for the spot volatility $v^{i}\left(B_{q}^{k} \mathbf{X}^{*}\right)$ we consider are

$$
\begin{aligned}
\operatorname{QRV}_{k, q}^{i}(\lambda) & =\frac{s\left(B_{q}^{k} \mathbf{X}^{*}, \lambda\right)}{v(\lambda)} \text { and } \\
\operatorname{MedRV}_{k, q}^{i} & =\frac{\operatorname{med}\left(\left|B_{q}^{k} \mathbf{X}^{*}\right|\right)^{2}}{\xi^{\operatorname{Med}}(k)}
\end{aligned}
$$

And likewise for $v^{j}\left(B_{q}^{k} \mathbf{X}^{*}\right)$.

\subsection{Estimation of the spot correlations}

The statistical literature on robust estimators for correlations is extensive (see e.g. Shevlyakov \& Smirnov (2011) and references therein). We measure spot correlations using benchmarks of this literature (Kendall's $\tau$ and Spearman's $\rho$ ), as well as other alternatives - quadrant signs and Gaussian ranks - that deliver good results in the realized literature (Boudt et al. (2012)).

To facilitate notations in this section, we denote $L_{q}=(q-1) k+1$ and $U_{q}=q k$ the lower and upper bounds for the index of returns belonging to $B_{q}^{k} \mathbf{X}^{*}$.

Kendall's $\tau$ is based on the statistical and geometric properties of elliptical distributions. Heuristically, it considers concordance of the combinations of observations by means of the signs. Kendall's $\tau$ between

\footnotetext{
${ }^{5}$ Boudt et al. (2012) use $k=5$ for the MedRV and a scaling of 1.624 .

${ }^{6}$ The constant 2.198 is the square of 1.483 , which is the scaling factor of the median absolute deviation in an i.i.d. Gaussian setup (Rousseeuw \& Croux (1993)).
} 
two random variables $X_{i}$ and $X_{j}$ is defined as

$$
\rho_{\tau}^{i, j}=E\left(\operatorname{sign}\left(X_{i}-\tilde{X}_{i}\right)\left(X_{j}-\tilde{X}_{j}\right)\right)
$$

where $\left(\tilde{X}_{i}, \tilde{X}_{j}\right)$ is an independent copy of $\left(X_{i}, X_{j}\right)$. In our setup, the estimator for block $q$ is

$$
r_{\tau, q}^{i, j}=\frac{2}{k(k-1)} \sum_{L_{q} \leq n<s \leq U_{q}} \operatorname{sign}\left(\left(\Delta_{n}^{N} X_{i}^{*}-\Delta_{s}^{N} X_{i}^{*}\right)\left(\Delta_{n}^{N} X_{j}^{*}-\Delta_{s}^{N} X_{j}^{*}\right)\right) .
$$

The estimated Pearson correlation is obtained as $r_{q}^{i, j}=\sin \left(\frac{\pi}{2} r_{\tau, q}^{i, j}\right)$. It is pairwise and does not necessarily provide positive definite estimated matrices. However, if the sample size is at least three times larger than the cross section, the resulting matrix is positive definite with probability one (Boudt et al. (2011a)).

Spearman's rho is based on the Pearson correlation between cumulative distribution functions. If one defines $F_{i}(x)=P\left(X_{i} \leq x\right)$ (and likewise for $\left.X_{j}\right)$, the Spearman's correlation is

$$
\rho_{S p}^{i, j}=\rho^{i, j}\left(F_{i}\left(X_{i}\right), F_{j}\left(X_{j}\right)\right) \text {. }
$$

The estimator for block $q$, denoted $r_{S p, q}^{i, j}$ is the sample Pearson correlation between ranks of the vectors in $B_{q}^{k} X^{*}$. The estimated Pearson correlation between $X_{i}$ and $X_{j}$ is then $r_{q}^{i, j}=2 \sin \left(\frac{\pi}{6} r_{S p, q}^{i, j}\right)$. The estimated matrix is positive definite with probability one if the sample size is at least two times larger than the cross sectional size (Boudt et al. (2011a)).

Quadrant signs, or quadrant correlations are defined as

$$
\rho_{Q d}^{i, j}=E\left(\operatorname{sign}\left(X_{1}-\operatorname{median}\left(X_{1}\right)\right)\left(X_{2}-\operatorname{median}\left(X_{2}\right)\right)\right) .
$$

The estimator based on $B_{q}^{k} X^{*}$ is the sample average of the signs

$$
r_{Q d, q}^{i, j}=\frac{1}{k} \sum_{n=L_{q}}^{U_{q}} \operatorname{sign}\left(\left(\Delta_{n}^{N} X_{i}^{*}-\operatorname{median}\left(\Delta_{n}^{N} X_{i}^{*}\right)\right)\left(\Delta_{n}^{N} X_{j}^{*}-\operatorname{median}\left(\Delta_{n}^{N} X_{j}^{*}\right)\right)\right) .
$$

The estimated Pearson correlations and the condition for positive definite estimated matrix are the same as for the Kendall's $\tau$.

Gaussian ranks is a direct estimator of the Pearson correlation:

$$
r_{\Phi, q}^{i, j}=\frac{1}{\psi_{k}} \sum_{n=L_{q}}^{U_{q}} \Phi^{-1}\left(\frac{\operatorname{rank}\left(\Delta_{n}^{N} X_{i}^{*}\right)}{k+1}\right) \Phi^{-1}\left(\frac{\operatorname{rank}\left(\Delta_{n}^{N} X_{j}^{*}\right)}{k+1}\right),
$$

where $\psi_{k}=\sum_{n=1}^{k} \Phi^{-1}\left(\frac{n}{k+1}\right)^{2}$ only depends on the amount of points $k$ in block $q, \Phi^{-1}(\cdot)$ denotes the quantile function of the standard normal distribution, and $\operatorname{rank}\left(\Delta_{n}^{N} X_{i}^{*}\right)$ the rank of $\Delta_{n}^{N} X_{i}^{*}$ in block $q$. Positive semi-definiteness is ensured as long as the sample size is greater than the cross section. 


\subsection{Positive definiteness and number of blocks}

The matrix version of (5) is

$$
\operatorname{DRC}(K)=\frac{1}{K} \sum_{q=1}^{K} \mathbf{S}\left(B_{q}^{k} X^{*}\right) \mathbf{R}\left(B_{q}^{k} X^{*}\right) \mathbf{S}\left(B_{q}^{k} X^{*}\right),
$$

where $\mathbf{R}(\cdot)$ denotes the jump robust estimator of the spot correlation matrix and $\mathbf{S}(\cdot)$ is a diagonal matrix containing jump robust estimates of the spot volatilities of each asset over block $q$.

Positive definiteness of $\operatorname{DRC}(K)$ depends on $\mathbf{R}(\cdot)$ and $\mathbf{S}(\cdot)$. If $\mathbf{R}(\cdot)$ is positive definite, and if the diagonal matrix $\mathbf{S}(\cdot)$ has no zero entries, then $\mathbf{S}(\cdot) \mathbf{R}(\cdot) \mathbf{S}(\cdot)$ and the average (9) are positive definite.

In practice choices have to be made for the number of blocks $K$ (and hence the number of observations per block $k$ ), which, for a fixed sample size $N$, lead to the classical trade-off between precision and bias. A smaller amount of blocks implies a larger amount of available observations for the estimation. In the opposite, estimation based on a larger amount of blocks allows to decrease the sensitivity to zero-returns, reducing the chances of downward biases. Moreover, the spot correlations and volatilities being time-varying, inference based on a small amount of blocks is unlikely to capture accurately the dynamics, advocating the use of a larger number of blocks.

Applied aspects must also be considered as different assets classes imply different trading hours. For instance, currencies trade continuously, commodities have several daylight trading breaks (e.g. corn traded at CBOT has a daylight trading break from 14:15 to 20:00), and equities typically trade from 9:30 to $16: 00$ (though some stock exchanges have lunch breaks). Trading hours therefore impact significantly the optimal sampling and blocking approaches. A second consideration is liquidity, as frequently and infrequently traded stocks belong both to the investment universe. Liquidity disparities greatly constrain the amount of blocks to use. Hautsch et al. (2012) proposed a liquidity-driven composite approach for realized kernels which tackles this problem.

Finally, the size of a considered portfolio imposes restrictions on the number of blocks. The amount of blocks per day should be a decreasing function of the cross section size $p$. In higher dimensions smaller amount of blocks should be used in order to preserve positive definiteness. Note that this points favors estimation of $\mathbf{R}(\cdot)$ with Gaussian ranks, which provide positive definite matrices as long as the amount of assets considered is smaller than the sample size. Many questions nevertheless remain open on this specific choice which heavily relies on the application. In ours, we use an ad hoc data-driven procedure for equities imposing each block to contain at least two times the size of the cross section, i.e. each block should have a minimum amount of point $k>2 p .^{7}$

\footnotetext{
${ }^{7}$ Synchronous prices are obtained from a calendar-time sampling approach, based on a data-driven sampling frequency choice, as described later in detail.
} 


\section{Market Microstructure noise}

Up to this point, we described the class of DRC estimators under the assumption that efficient prices are observable and hence they are not contaminated by market microstructure noise. In practice, however, prices do not always match with those that are exchanged on markets (see e.g. Zhou (1996)). From a modeling viewpoint, noise is an additive component to log-prices that can be written as $\mathbf{Y}_{t}=\mathbf{X}_{t}+\boldsymbol{\eta}_{t}$, where $\mathbf{X}_{t}$ denotes the efficient log-prices (1) and $\boldsymbol{\eta}_{t}$ denotes the market microstructure noise. Generally, noise generates upward biases in standard realized volatilities, though it impact less covariance measures. In the sequel we describe three techniques for decreasing the influence of noise.

Sparse sampling consists of sampling prices on a sparse grid of time points, i.e. choosing a value for $\Delta$ in the interpolation scheme. At lower frequencies, the impact of noise is known to be less relevant and the bias tends to vanish (Barndorff-Nielsen \& Shephard (2007)). The resulting estimator is computed using the synchronous low frequency data. Sparse sampling may reduce significantly the number of available observations, which has two drawbacks: it limits the size of the cross section for which the estimated matrix is positive definite, and the precision of the estimates worsens.

Subsampling is introduced in the univariate case by Zhang et al. (2005) and Zhang (2006), and studied in the multivariate setup by Zhang (2011) and Boudt \& Zhang (2013) among others. Instead of using contiguous non-overlapping blocks of returns, subsampling uses overlapping subsamples containing returns sampled at a lower frequency. The successive estimates are then averaged.

McAleer \& Medeiros (2008) provide the following intuitive example. If one has noisy 1-second returns and wishes to use subsampling with 5-minute returns, she can compute returns using log-prices recorded on the following grid of time coordinates [9:30-9:35], [9:35-9:40], [9:40-9:45], ... , [15:55-16:00]. The unused times are used to construct new series of returns. For example, starting 10 seconds later the new grid is [9:30:10-9:35:10], [9:35:10-9:40:10], [9:40:10-9:45:10], ... , [15 50:10-15:55:10]. The new series are used to replace blocks and estimate the covariance.

We construct $K$ overlapping sub-grids at a calendar frequency $\delta$, i.e. we skip $\delta$ points in $\pi_{N_{i}}$ between two consecutive points of the new sub-grid. ${ }^{8}$ Returns are computed from prices projected on the sparse grid of times using previous-tick interpolation. They are denoted by $\Delta_{m, q}^{N} \mathbf{Y}^{*}=\sqrt{N / \delta}\left(\mathbf{Y}_{\frac{(q-1)+\delta m}{N}}-\mathbf{Y}_{\frac{(q-1)+\delta(m-1)}{N}}\right)$ where $q=1, \ldots, K, m=1, \ldots,\lfloor(N-q+1) / \delta\rfloor$, and $\lfloor$.$\rfloor denotes the floor operator. { }^{9}$ We denote the new set of returns contained in subsample $q$ by $B_{q}^{\delta} \mathbf{Y}^{*}=\left(\Delta_{m, q}^{N} \mathbf{Y}^{*}\right)_{1 \leq m \leq\lfloor(N-q+1) / \delta\rfloor}$. The estimator is then computed as in (5) by replacing the blocks by the new subsamples.

Sampling at lower frequencies allows to decrease the impact of noise on estimates, and averaging over the subsamples allows to increase the efficiency of the estimator. However, the cost of subsampling is that

\footnotetext{
${ }^{8}$ For emphasizing that the subsampling and blocks are mutually exclusive, deliberately we use the same notation for both techniques.

${ }^{9}$ Note that if returns are sampled every second along a grid $\pi_{N_{i}}$ of type 1 , then $K=\delta$.
} 
the size of the cross section for which the estimator is positive definite will be limited by the frequency of the subsamples. The reason is alike to sparse sampling since the average is composed of covariance matrices based on low frequency data.

Pre-averaging is introduced by Podolskij \& Vetter (2009) and studied by Jacod et al. (2009) -multivariate extensions can be found in Christensen et al. (2010a) and Christensen et al. (2013). Pre-averaging relies on the intuitive idea that if the noise $\boldsymbol{\eta}_{t}$ is i.i.d. with mean zero, then smoothing the log-prices $\mathbf{Y}_{t}$ may decrease the impact of microstructure noise and provide an approximation of the true latent price $\mathbf{X}_{t}$.

We use pre-averaging in calendar time assuming prices are aligned on an homogeneous grid of time. If $\Delta_{m}^{N} \mathbf{Y}^{*}$ denotes the $m$-th vector of noisy scaled returns, pre-averaged returns are

$$
\Delta_{m} \tilde{\mathbf{Y}}^{*}=\frac{1}{\sqrt{k_{N} \psi}} \sum_{j=1}^{k_{N}-1} g\left(\frac{j}{k_{N}}\right) \Delta_{m+j}^{N} \mathbf{Y}^{*}, \text { for } m=0, \ldots, N-k_{N}+1,
$$

where $g($.$) is a kernel function, \psi=\frac{1}{k_{N}} \sum_{j=1}^{k_{N}-1} g^{2}\left(\frac{j}{k_{N}}\right)$ and $k_{N} / \sqrt{N}=\theta+o\left(N^{-1 / 4}\right)$ are computed following Christensen et al. (2010a). The scaling in front of the sum in (10) is necessary to avoid in-sample biases of the estimates. The pre-averaged version of the class of DRC is provided by replacing the noisy returns used to construct the blocks in the previous section by their pre-averaged counterpart $\Delta_{m} \tilde{\mathbf{Y}}^{*}$, and compute the estimator following (5).

Pre-averaging of log-returns allows to keep more points than sparse sampling for the estimation. Consequently, precise estimates can be obtained without reducing the dimension. As pointed out by Christensen et al. (2010a), there is a strong relationship between pre-averaging and subsampling. Estimators relying on these techniques can be mapped into each other. By using one of these techniques to compute the cumulative squared returns, the estimators use all the data points, but put slightly different weights on them, which explains the differences found in the next sections.

\section{Synchronization schemes}

We now assume that the partitions $\pi_{N_{1}}$ and $\pi_{N_{2}}$ are of type 3 or 4 , i.e. observations are asynchronous and assets may have different sample sizes. Asynchronous trading induces microstructure effects that can lead to downward bias in the correlations among assets, such as the Epps effect (after Epps (1979)) that is illustrated in Figure 2. It shows the baseline realized correlations (of Andersen et al. (2003)) between Apple and the SPDR S\&P500 ETF on April 302012 and as a function of the sampling frequency. The correlation increases with the sampling interval and reach a stable level for low sampling frequencies.

As in the synchronous case, every day there is an opening and a closing, and the day is evenly divided in $K$ blocks. The main difference with regularly spaced arrivals is that the number of observations per block is random. Once re-scaled, these observations can be used to estimate the volatilities, one by one independently of each other. However, for the estimation of the correlations within each block, observations for all assets need to be synchronized. What follows is the list of the synchronization techniques 


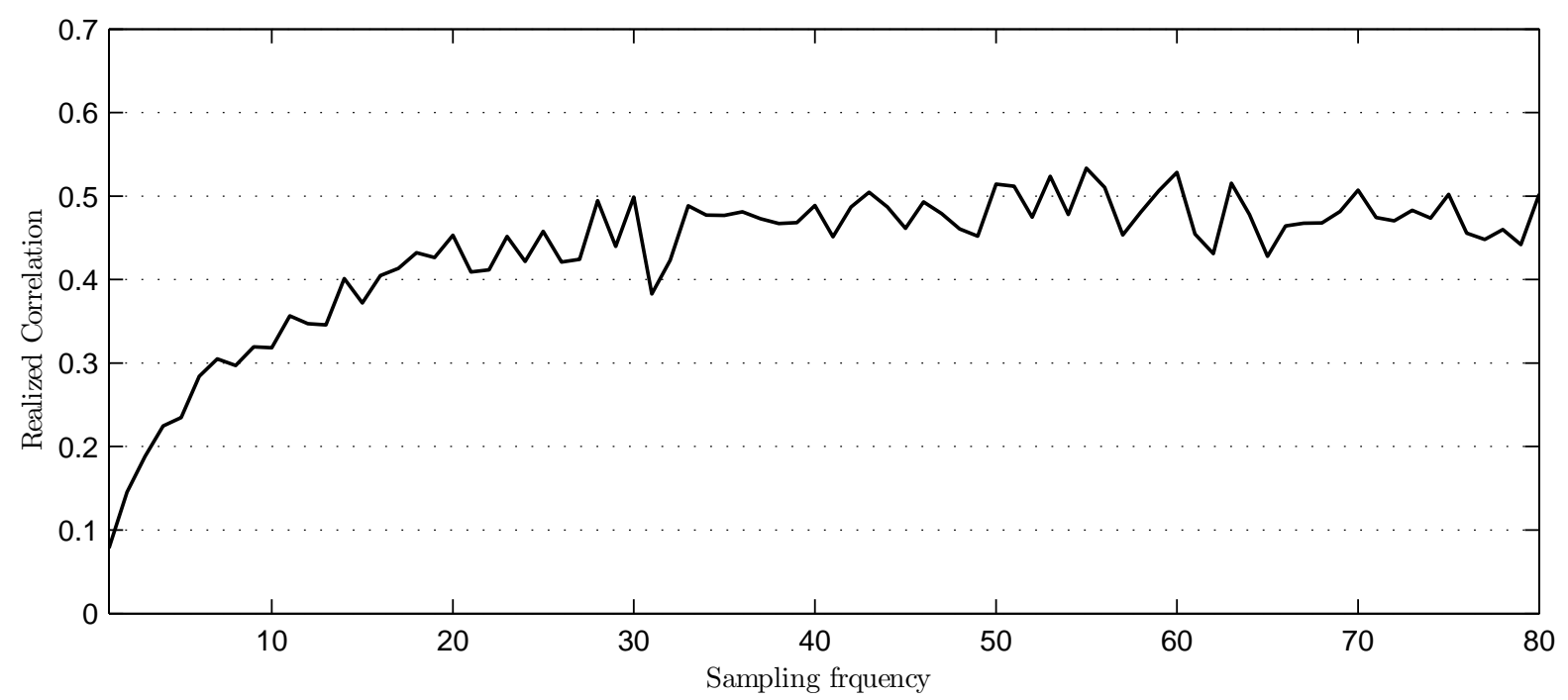

Figure 2: This figure shows an example of microstructure effects induced by asynchronous trading on correlations between SPY and APPL. The x-axis represents the calendar sampling frequency in seconds.

that we use.

Interpolation is based on first choosing a fixed calendar sampling frequency. Indeed, it is common in practice to compute returns on basis of prices sampled every 5,10 and 15 minutes. This is the multivariate extension of sparse sampling (see Andersen et al. (2001)) and it provides homogenous time series (Dacorogna et al. (2001)). The sampling frequency is chosen according to an optimality criterion, such as minimizations related to market microstructure noise and jumps.

The choice of the calendar sampling frequency is delicate and may significantly modify the statistical properties of the estimators. On the one hand, sampling at higher frequencies entails a larger sample and potentially more precise estimates. On the other hand, the impact of microstructure noise is more important at high frequencies.

Once the frequency is chosen, homogeneous price vectors are constructed. If we denote by $\Delta t$ the frequency at which we sample returns, the number of observations is $\lfloor$ day length (seconds) $/ \Delta t$ (seconds) $\rfloor$. Then, if one denotes by $t_{0}$ the starting time of the day, the $i$-th observation is constructed as $\mathbf{X}_{t_{i}}=\mathbf{X}_{t_{k}}$ where $k=\max \left(\tilde{k} \mid t_{\tilde{k}} \leq t_{0}+i \Delta t\right)$ and $t_{k} \leq t_{0}+i \Delta t \leq t_{k+1}$. I.e. prices are constructed by projecting the closest past observation to the $i$-th point of the time grid. This interpolation is called previous tick.

Alternatively, one may opt for linear interpolation. The construction is similar to the previous technique and the amount of points in the homogeneous vector is deterministic. If one denotes by $t_{0}$ the starting time of the day, the $i$-th observation is constructed as

$$
\mathbf{X}_{t_{i}}=\mathbf{X}_{t_{k}}+\frac{t_{0}+i \Delta t-t_{k}}{t_{k+1}-t_{k}}\left(\mathbf{X}_{t_{k+1}}-\mathbf{X}_{t_{k}}\right)
$$

where $k=\max \left(\tilde{k} \mid t_{\tilde{k}} \leq t_{0}+i \Delta t\right)$. 
Refresh time. Barndorff-Nielsen et al. (2011) construct vectors of homogeneous high-frequency prices by projecting asynchronous data on a grid of time coordinates that has a random amount of data depending on the relative trading intensity/liquidity of the assets considered. As a result, the less liquid asset drives the construction of the grid. A similar approach was previously used by Harris et al. (1995) and Martens (2004).

Refresh time can in fact be seen as a previous-tick interpolation on a grid of time coordinates defined as follows.

Definition 2 Denote the trading times of an asset $i$ as $t_{1}^{i}, t_{2}^{i}, \ldots$ for $i=1, \ldots, p$ and let $N_{i}^{t}$ be the amount of trades recorded up to time $t$ ( with $N_{i}^{1}=N_{i}$ ). Then, the first refresh time is defined as $\tau_{1}=\max \left(t_{1}^{1}, \ldots, t_{1}^{p}\right)$ and the subsequent refresh times as $\tau_{j+1}=\max \left(t_{N_{1}^{1}+\tau_{j}}^{\tau_{j}}, \ldots, t_{N_{p} \tau_{j}+1}^{p}\right)$.

The time $\tau_{1}$ designates the first moment at which all the assets are traded at least once, i.e. the first time at which all prices are refreshed. Then, $\tau_{2}=\max \left(t_{N_{1}^{\tau_{1}}+1^{1}}^{1}, \ldots, t_{N_{p}^{\tau_{1}+1}}^{p}\right)$ and, from $\tau_{1}$, we have that $N_{i}^{\tau_{1}} \geq 1 \forall i \in\{1,2, \ldots, p\}$. Moreover, if $\max \left(t_{1}^{1}, \ldots, t_{1}^{p}\right)=t_{1}^{i}$ then $i$ is such that $N_{i}^{t_{1}^{i}}=1$. Thus, we have that $N_{i}^{\tau_{1}}+1 \geq 2 \forall i \in\{1,2, \ldots, p\}$ and $\min \left(N_{1}^{\tau_{1}}+1, \ldots, N_{p}^{\tau_{1}}+1\right)=2$. Intuitively $\tau_{2}$ is the first time after $\tau_{1}$ at which all the assets are traded again. This can be recursively applied for $\tau_{3}, \tau_{4}, \ldots$ up to the end of the sample. The sample size after synchronization is random and large cross sections induce more complex computations, increasing the risk of deleting a lot of observations.

Refinements have been proposed in the literature. Fan et al. (2012) use a pairwise version of refresh time ("pairwise-refresh" as opposed to "all-refresh"). This approach has the advantage of retaining more observations. However, it does not provide semi-positive definite matrices, as pointed out by Fan et al. (2012). Hautsch et al. (2012) rank stocks according to their relative liquidity and apply refresh-time to estimate high-dimensional realized kernels, coupled with blocking and regularization techniques related to random matrix theory.

Hayashi \& Yoshida (2005)'s scheme handles asynchronous data without projecting prices. As a result, all prices are used in the computation of realized covariances. This scheme was first used to compute realized covariances by aggregating returns recorded in overlapping time intervals. The estimator of cumulative covariance between assets $i$ and $j$ is defined as follows:

$$
\mathbf{R C}_{H Y}^{i, j}=\sum_{m=1}^{N_{i}} \sum_{n=1}^{N_{j}} \Delta_{i, m}^{N_{i}} \mathbf{X} \Delta_{j, n}^{N_{j}} \mathbf{X} \mathbf{1}_{\left\{\left(t_{m-1}^{i}, t_{m}^{i}\right] \cap\left(t_{n-1}^{j}, t_{n}^{j}\right] \neq \varnothing\right\}^{\prime}}
$$

where $\mathbf{1}_{\left\{\left(t_{m-1}^{i}, t_{i}^{i}\right] \cap\left(t_{n-1}^{j}, t_{n}^{j}\right] \neq \varnothing\right\}}$ is an indicator function that takes value 1 is the argument is true. This aggregation scheme can be used with different versions of the cumulative covariance estimator as, for example, the thresholds realized covariances of Mancini \& Gobbi (2012). However, the class of DRC does not map naturally into the construction of aggregated returns and hence, in our comparison below, we 
only apply it to the realized covariances of Andersen et al. (2003) and the thresholds realized covariances of Mancini \& Gobbi (2012). More details are provided in the next section.

\section{Monte Carlo Simulation}

We assess the finite sample behavior of the combinations of realized volatilities, correlations and synchronization techniques. We report the performances and compare the best combinations with five competing estimators: the baseline realized covariance (RC henceforth) of Andersen et al. (2003), realized bi-power covariation (BPC) of Barndorff-Nielsen \& Shephard (2004a), realized outlyingness weighted covariance (OWC) of Boudt et al. (2011b), the estimators based on thresholds (TC) of Mancini \& Gobbi (2012), and realized kernels (RK) of Barndorff-Nielsen et al. (2011).

We implement these estimators as suggested by the authors. For instance, TC is estimated with a hreshold value $r_{h, t}=9 \mathrm{BPV}_{t} \Delta_{t}^{0.98}$, following Jacod \& Todorov (2009), where $\mathrm{BPV}_{t}$ is the bipower variation and $\Delta_{t}$ refers to the time interval between two successive returns. We implement OWC with hard rejection functions and a threshold of 0.999, following the results of Boudt et al. (2011b). As for RK, we use refresh time and subsampled realized variances to compute the optimal bandwidth. When necessary more details are given below.

In a nutshell, the conclusion of our study is that across the four simulated models (presented below) with jumps, noise and asyncrhonous prices, pre-averaged DRC implemented with Gaussian ranks provides the best results.

\subsection{Data generating processes}

We simulate 10000 trading days from four models that are often used in the literature: a Brownian motion, the Heston model, a stochastic volatility with constant correlation, and a continuous GARCH diffusion. Assuming that the market opens 252 days per year and 6.5 hours per day, a trading day has 23400 seconds. This is the number of prices we generate per day (using the Euler discretization scheme) and that implies that 1 second corresponds to $1 /(252 \times 23400)$ units of time.

In the sequel of this section we first show the models used to generate the continuous part $X^{c}$. Simulation is done following the choices made in previous works (see Table 1). Next, results are divided in four sub-sections. We start with the ideal world where assets trade synchronously and without noise (section 5.2). Then we introduce asynchronicity (5.3), noise (5.4), and asynchronicity and noise (5.5). ${ }^{10}$

Model 1 is a Brownian motion with constant parameters:

$$
d X_{i t}^{c}=\sigma_{i} d W_{i t}
$$

for $i=1,2$, and where $W_{i t}$ are Brownian motions (also denoted by $B_{i t}$ in the next models) and $<d W_{1 t}, d W_{2 t}>=\rho d t$. The initial $\log$ prices are $X_{1,0}=\log (100)$ and $X_{2,0}=\log (40)$.

\footnotetext{
${ }^{10}$ We show results for RK for the two cases with noise.
} 
Table 1: Parameter choices

\begin{tabular}{|c|c|c|c|c|c|c|c|c|c|}
\hline \multicolumn{10}{|c|}{ Model 1 - Standard Brownian Motion } \\
\hline & $\sigma_{i}$ & $\rho$ & & & & & & & \\
\hline $\mathrm{i}=1$ & 0.15 & 0.3 & & & & & & & \\
\hline$i=2$ & 0.45 & & & & & & & & \\
\hline \multicolumn{10}{|c|}{ Model 2 - Ait-Sahalia et al. (2010) } \\
\hline & $\mu_{i}$ & $\kappa_{i}$ & $\lambda_{i}$ & $\bar{\sigma}_{i}^{2}$ & $s_{i}$ & $\theta_{i}$ & $\eta_{i}$ & $\rho_{i}$ & $\rho$ \\
\hline $\mathrm{i}=1$ & 0.05 & 3 & 12 & 0.16 & 0.8 & -5 & 0.8 & -0.6 & 0.5 \\
\hline$i=2$ & 0.03 & 2 & 36 & 0.09 & 0.5 & -6 & 0.5 & -0.75 & \\
\hline \multicolumn{10}{|c|}{ Model 3 - Barndorff-Nielsen et al. (2011) } \\
\hline & $\mu_{i}$ & $\beta_{0 i}$ & $\beta_{1 i}$ & $\alpha_{i}$ & $\rho_{i}$ & & & & \\
\hline $\mathrm{i}=1$ & 0.03 & $-5 / 6$ & $1 / 8$ & $-1 / 40$ & 0.5 & & & & \\
\hline$i=2$ & 0.03 & $-5 / 6$ & $1 / 8$ & $-1 / 40$ & 0.5 & & & & \\
\hline \multicolumn{10}{|c|}{ Model 4 - Voev \& Lunde (2007) and Andersen \& Bollerslev (1998) } \\
\hline & $k_{i}$ & $\theta_{i}^{2}$ & $\omega_{i}$ & $\sigma_{i, 0}^{2}$ & $k_{x}$ & $\theta_{x}$ & $\omega_{x}$ & $\rho_{0}$ & \\
\hline $\mathrm{i}=1$ & 0.35 & 0.636 & 0.296 & 0.64 & 0.03 & 0.64 & 0.118 & 0.5 & \\
\hline$i=2$ & 0.35 & 0.636 & 0.296 & 0.16 & & & & & \\
\hline
\end{tabular}

Model 2 is the Heston model, in which correlations remain constant while volatilities change over time and display jumps, as in Ait-Sahalia et al. (2010) and Shephard \& Xiu (2012). For $i=1$, 2, we simulate log-prices as

$$
\begin{aligned}
d X_{i t}^{c} & =\mu_{i} d t+\sigma_{i t} d W_{i t} \\
d \sigma_{i t}^{2} & =\kappa_{i}\left(\bar{\sigma}_{i}^{2}-\sigma_{i t}^{2}\right) d t+s_{i} \sigma_{i t} d B_{i t}+\sigma_{i t-} J_{i t}^{V o l} d N_{i t},
\end{aligned}
$$

where $<d W_{i t}, d B_{j t}>=\delta_{i j} \rho_{i} d t$ ( $\delta_{i j}$ denotes the Kronecker delta), $<d W_{1 t}, d W_{2 t}>=\rho d t$, and $\kappa_{i}>0$. The model is calibrated as in Aitt-Sahalia et al. (2010). For each new path we generate a starting value for $\sigma_{i t_{0}}^{2}$ from a Gamma distribution $\Gamma\left(2 \kappa_{i} \bar{\sigma}_{i}^{2} / s_{i}^{2}, s_{i}^{2} / 2 \kappa_{i}\right)$, the jump size $\log J_{i t}^{V o l}$ is distributed like $N\left(\theta_{i}, \eta_{i}\right)$, $N_{i t}$ is a Poisson Process with parameter $\lambda_{i}$, and initial log-prices are set equal to $X_{1,0}=\log (100)$ and $X_{2,0}=\log (40)$.

Model 3 also has stochastic volatility and constant correlation. It follows the model on Barndorff-Nielsen et al. (2011), used for assessing the finite sample properties of multivariate realized kernels. For $i=1,2$, we simulate log-prices as

$$
\begin{aligned}
d X_{i t}^{c} & =\mu_{i} d t+d V_{i t}+d F_{i t} \\
d V_{i t} & =\rho_{i} \sigma_{i t} d B_{i t} \\
d F_{i t} & =\sqrt{1-\rho_{i}^{2}} \sigma_{i t} d W_{t} \\
\sigma_{i t} & =\exp \left(\beta_{i 0}+\beta_{i 1} \zeta_{i t}\right) \\
d \zeta_{i t} & =\alpha_{i} \zeta_{i t} d t+d B_{i t},
\end{aligned}
$$

where $<d W_{t}, d B_{j t}>=0,<d X_{1 t}, d X_{2 t}>=\sqrt{1-\rho_{1}^{2}} \sqrt{1-\rho_{2}^{2}} d t$. We calibrate the model following 
Barndorff-Nielsen et al. (2011).

Model 4 is a continuous GARCH diffusion. This is the only model allowing stochastic correlations and volatilities. For $i=1,2$, we simulate $\log$-prices as

$$
\begin{aligned}
d X_{i t}^{c} & =\sigma_{i t} d W_{i t} \\
d \sigma_{i t}^{2} & =\kappa_{i}\left(\theta_{i}^{2}-\sigma_{i t}^{2}\right) d t+\omega_{i} \sigma_{i t}^{2} d B_{i t} \\
d x_{t} & =\kappa_{x}\left(\theta_{x}-x_{t}\right) d t+\omega_{x} x_{t} d B_{x t} \\
\rho_{t} & =\left(e^{2 x_{t}}-1\right) /\left(e^{2 x_{t}}+1\right),
\end{aligned}
$$

where $<d W_{i t}, d B_{j t}>=0,<d W_{1 t}, d W_{2 t}>=\rho_{t} d t$. We calibrate the parameters following Voev \& Lunde (2007) and Andersen \& Bollerslev (1998).

Jumps, noise, asynchronous trading, and accuracy measure. To allow for co-jumps we simulate three independent compound Poisson processes. The two first processes correspond to the individual jump activity while the third process is common to both assets. We simulate jump arrivals with Poisson processes, and jumps sizes with i.i.d. log-normally distributed random variables. The expected amount of jumps per day corresponds to the parameter of the Poisson processes and are set to 2 for the individual jump activities and 4 for the common jump process. I.e. assets are expected to jump 6 times per day. Jumps sizes are simulated from i.i.d. $N(0, \zeta)$ where $\zeta=\frac{0.7}{252}$.

We simulate noise as in Barndorff-Nielsen et al. (2008) assuming that $\eta_{t}^{i} \sim$ i.i.d. $N\left(0, \omega_{i}^{2}\right)$ where $\omega_{i}^{2}=\xi^{2} \sqrt{N^{-1} \sum_{m=1}^{N} \sigma_{m / N}^{i, 4}}$ and $\xi^{2}=0.01$.

Non-synchronous trading is introduced using Bernoulli trials. This technique, based on Aït-Sahalia et al. (2010), selects randomly prices from a grid of evenly spaced transactions. One difference in our scheme is that we simulate probability levels from a uniform distribution over $[0.25,1]$. It enables to cover various regimes of relative liquidity between assets and to test the goodness of our estimators independently from a fixed trading intensity.

Last, to compare the finite sample performances of the estimators we use the root mean squared relative errors (as in Boudt et al. (2011b)):

$$
\operatorname{RMSE}=\sqrt{\frac{1}{T k} \sum_{t=1}^{T}\left\|\operatorname{vech}\left(\operatorname{Estim}_{t}-\mathbf{I C}_{t}\right) \cdot / \operatorname{vech}\left(\mathbf{I C}_{t}\right)\right\|^{2}},
$$

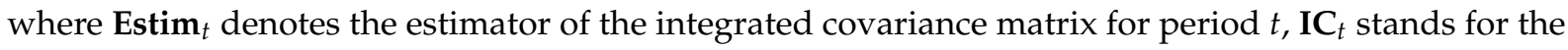
integrated covariance matrix for period $t, . /$ denotes the element wise division, and $T$ is the amount of simulated periods (days). 


\subsection{Synchronous prices \& no noise}

Table 2 reports the results. It is divided in four panels, corresponding to different intraday sampling frequencies (30 seconds, and 1, 5, and 15 minutes). Each panel shows the RMSE for eight estimators. The first four are the competitors (RC, BPC, TC and OWC) while the last four are our combinations, which we denote Med-Ken, Med-Spear, Med-Quad and Med-Gauss (for instance Med-Spear stands for the MedRV estimator for the volatilities and Spearman's $\rho$ for the correlations). Column-wise, the table is divided in the four models, with and without jumps. In the interest of space the QRV estimators are not shown, as MedRV is uniformly better (results are nevertheless reported in Table 7 of the Appendix).

Four are the main findings. First, not surprisingly, RC performs well without jumps but it is very sensitive to them at high frequencies. The other estimators provide better performance in presence of jumps, as they are robust to them. Second, in general the quality of the estimators decreases with the sampling frequency. Lower frequencies provide less precise estimates. On average, the relative accuracy of the estimators for 1-, 5- and 15-minute returns compared to the RMSE of estimators computed on basis of 30-second returns are of order close to $\sqrt{2}, \sqrt{10}$ and $\sqrt{30}$ respectively. Similar results are found by Boudt et al. (2011b). ${ }^{11}$

Third, the threshold used for TC provides good results, as the performances of TC are close to those of RC in absence of jumps and far better when jumps are added. ${ }^{12}$ Moreover, most of the time OWC outperforms the other estimators, followed by TC for high frequencies. Our combinations are generally less accurate than OWC and TC and perform on average better than the BPC. The goodness of our estimators will appear more clearly in case of asynchronous trading.

Fourth, and as briefly mentioned above, combinations based on median operators for volatility measures provide in general more accurate measures than those based on quantiles. Moreover, quadrant correlations perform in a less convincing way than Gaussian ranks, Spearman's $\rho$ and Kendall's $\tau$. In the sequel we only show results for the combination based on median measures for volatilities and Spearman's $\rho$ and Gaussian ranks for correlations. We skip Kendall's $\tau$ as it is computationally less efficient than the others.

\subsection{Asynchronous prices \& no noise}

Next, we study the impact of asynchronous trading. Following Mancini \& Gobbi (2012), RC and TC are implemented with the aggregation scheme proposed by Hayashi \& Yoshida (2005); we denote this estimator as HY-RC and HY-TC. For OWC and BPC, we follow Boudt et al. (2011b) and use returns aligned on a 5-minute grid with previous-tick interpolation in order to avoid microstructure effects related to asynchronous trading; we denote the estimators PT-BPC and PT-OWC. Our estimators are computed with data synchronized using refresh time (denoted RT), 30-seconds previous tick (denoted PT) and 30-seconds linear interpolation (denoted LI).

\footnotetext{
${ }^{11}$ Our model 3 corresponds to Boudt et al. (2011b)'s main model, except that the jump process is different.

${ }^{12} \mathrm{TC}$ is a truncated version of RC and hence should provide equivalent results in absence of jumps
} 
Table 2: Monte Carlo study - Synchronous trading.

\begin{tabular}{|c|c|c|c|c|c|c|c|c|}
\hline & \multicolumn{2}{|c|}{ Model 1} & \multicolumn{2}{|c|}{ Model 2} & \multicolumn{2}{|c|}{ Model 3} & \multicolumn{2}{|c|}{ Model 4} \\
\hline & no jumps & jumps & no jumps & jumps & no jumps & jumps & no jumps & jumps \\
\hline \multicolumn{9}{|c|}{ Panel A: 30-seconds returns } \\
\hline $\mathrm{RC}$ & 0.082 & 24.05 & 0.946 & 9.421 & 0.054 & 3.950 & 0.062 & 3.353 \\
\hline $\mathrm{BPC}$ & 0.100 & 1.622 & 0.943 & 1.583 & 0.062 & 0.341 & 0.073 & 0.399 \\
\hline TC & 0.086 & 0.085 & 0.918 & 0.961 & 0.060 & 0.058 & 0.067 & 0.065 \\
\hline OWC & 0.084 & 0.087 & 0.936 & 0.970 & 0.056 & 0.056 & 0.064 & 0.065 \\
\hline Med-Ken & 0.105 & 0.114 & 0.954 & 1.007 & 0.083 & 0.084 & 0.090 & 0.092 \\
\hline Med-Spear & 0.105 & 0.113 & 0.955 & 1.008 & 0.083 & 0.084 & 0.090 & 0.092 \\
\hline Med-Quad & 0.131 & 0.134 & 0.955 & 1.008 & 0.087 & 0.088 & 0.100 & 0.101 \\
\hline Med-Gauss & 0.103 & 0.137 & 0.953 & 1.007 & 0.082 & 0.083 & 0.088 & 0.091 \\
\hline \multicolumn{9}{|c|}{ Panel B: 1-minute returns } \\
\hline $\mathrm{RC}$ & 0.116 & 24.04 & 0.954 & 9.414 & 0.076 & 3.955 & 0.089 & 3.352 \\
\hline $\mathrm{BPC}$ & 0.140 & 2.210 & 0.953 & 1.930 & 0.087 & 0.495 & 0.103 & 0.556 \\
\hline $\mathrm{TC}$ & 0.120 & 0.123 & 0.924 & 0.972 & 0.083 & 0.083 & 0.094 & 0.092 \\
\hline OWC & 0.120 & 0.121 & 0.943 & 0.977 & 0.079 & 0.081 & 0.092 & 0.092 \\
\hline Med-Ken & 0.149 & 0.173 & 0.955 & 1.037 & 0.117 & 0.122 & 0.125 & 0.132 \\
\hline Med-Spear & 0.150 & 0.169 & 0.957 & 1.040 & 0.116 & 0.122 & 0.125 & 0.133 \\
\hline Med-Quad & 0.184 & 0.193 & 0.958 & 1.039 & 0.121 & 0.127 & 0.138 & 0.146 \\
\hline Med-Gauss & 0.146 & 0.210 & 0.953 & 1.036 & 0.116 & 0.121 & 0.123 & 0.131 \\
\hline \multicolumn{9}{|c|}{ Panel C: 5-minute returns } \\
\hline $\mathrm{RC}$ & 0.258 & 24.09 & 0.973 & 9.473 & 0.168 & 3.957 & 0.197 & 3.368 \\
\hline $\mathrm{BPC}$ & 0.309 & 5.485 & 0.970 & 3.347 & 0.194 & 1.128 & 0.226 & 1.175 \\
\hline $\mathrm{TC}$ & 0.263 & 0.918 & 0.944 & 1.371 & 0.179 & 0.300 & 0.205 & 0.344 \\
\hline OWC & 0.269 & 0.293 & 0.951 & 1.114 & 0.177 & 0.212 & 0.205 & 0.240 \\
\hline Med-Ken & 0.334 & 0.582 & 1.034 & 1.356 & 0.253 & 0.326 & 0.275 & 0.371 \\
\hline Med-Spear & 0.340 & 0.577 & 1.041 & 1.372 & 0.253 & 0.331 & 0.277 & 0.383 \\
\hline Med-Quad & 0.402 & 0.556 & 1.044 & 1.360 & 0.264 & 0.333 & 0.304 & 0.390 \\
\hline Med-Gauss & 0.328 & 0.637 & 1.029 & 1.352 & 0.252 & 0.323 & 0.274 & 0.367 \\
\hline \multicolumn{9}{|c|}{ Panel D: 15-minute returns } \\
\hline $\mathrm{RC}$ & 0.443 & 24.10 & 1.025 & 9.380 & 0.291 & 3.983 & 0.335 & 3.421 \\
\hline $\mathrm{BPC}$ & 0.521 & 9.391 & 1.010 & 4.830 & 0.333 & 1.773 & 0.380 & 1.797 \\
\hline $\mathrm{TC}$ & 0.448 & 5.839 & 0.992 & 3.551 & 0.310 & 1.230 & 0.349 & 1.337 \\
\hline OWC & 0.479 & 2.986 & 0.972 & 2.404 & 0.327 & 0.773 & 0.371 & 0.866 \\
\hline Med-Ken & 0.582 & 1.504 & 1.202 & 2.302 & 0.451 & 0.720 & 0.481 & 0.874 \\
\hline Med-Spear & 0.591 & 1.525 & 1.207 & 2.336 & 0.452 & 0.729 & 0.483 & 0.895 \\
\hline Med-Quad & 0.688 & 1.367 & 1.238 & 2.323 & 0.467 & 0.743 & 0.523 & 0.917 \\
\hline Med-Gauss & 0.571 & 1.656 & 1.193 & 2.304 & 0.447 & 0.713 & 0.474 & 0.871 \\
\hline
\end{tabular}

Table 2 reports Monte Carlo simulation results for eight estimators of the integrated covariance matrix under four different models, specified with and without jumps. The entries report the root mean square errors (RMSE) computed as in (17) for 10000 draws of 23400 observations. Prices are simulated simultaneously each second and without noise. Every panel of the table contains the four competitors (RC, BPC, TC and OWC), followed by the disentangled estimators (Med-Ken, Med-Spear, Med-Quad and Med-Gauss), which are computed with 5 blocks (except for 15-minute returns for which only 1 block is used). 
Results are in Table 3 from which we draw two conclusions. First, estimators based on the HayashiYoshida scheme are quite good. HY-TC is the most efficient way to estimate the integrated covariance matrix. LT-OWC losses efficiency because of the lower sampling frequency. Nevertheless, at higher frequencies, biases related with the Epps effect appear, as discussed in Boudt et al. (2011b).

Second, our estimators perform on average less efficiently than HY-TC. The 30-second linear interpolation scheme leads to the best results among the different combinations for disentangled realized covariances. Without jumps it performs less efficiently than HY-RC but better than other estimators. In presence of jumps it performs better than all its competitors except for HY-TC. This result, though unfavorable for the class of disentangled realized measures, is logical. Hayashi-Yoshida scheme uses all the data points by aggregating returns that have been recorded on overlapping time periods. As mentioned previously, interpolation techniques project prices on fixed grids and inevitably delete data points, just as refresh time and other generalized synchronization schemes.

Table 3: Monte Carlo - Asynchronous trading and no noise.

\begin{tabular}{lcccccccc}
\hline \hline & \multicolumn{2}{c}{ Model 1 } & \multicolumn{2}{c}{ Model 2 } & \multicolumn{2}{c}{ Model 3 } & \multicolumn{2}{c}{ Model 4 } \\
& no jumps & jumps & no jumps & jumps & no jumps & jumps & no jumps & jumps \\
\hline HY-RC & 0.030 & 17.08 & 0.934 & 6.874 & 0.018 & 2.755 & 0.021 & 2.329 \\
PT-BPC & 0.317 & 4.055 & 0.971 & 2.714 & 0.191 & 0.813 & 0.225 & 0.923 \\
HY-TC & 0.040 & 0.034 & 0.905 & 0.926 & 0.031 & 0.028 & 0.034 & 0.030 \\
PT-OWC & 0.274 & 0.292 & 0.948 & 1.100 & 0.176 & 0.211 & 0.202 & 0.247 \\
PT-Spear & 0.078 & 0.101 & 0.926 & 0.945 & 0.039 & 0.041 & 0.051 & 0.049 \\
LI-Spear & 0.072 & 0.092 & 0.931 & 0.950 & 0.028 & 0.030 & 0.042 & 0.042 \\
RT-Spear & 0.066 & 0.068 & 0.920 & 0.938 & 0.062 & 0.062 & 0.064 & 0.062 \\
PT-Gauss & 0.076 & 0.136 & 0.925 & 0.946 & 0.040 & 0.044 & 0.051 & 0.048 \\
LI-Gauss & 0.069 & 0.128 & 0.930 & 0.951 & 0.028 & 0.031 & 0.041 & 0.040 \\
RT-Gauss & 0.066 & 0.075 & 0.920 & 0.938 & 0.063 & 0.063 & 0.064 & 0.062 \\
\hline \hline
\end{tabular}

Table 3 reports Monte Carlo simulation results for eight estimators of the integrated covariance matrix under four different models, specified with and without jumps. The entries report the root mean square errors (RMSE) computed as in (17) for 10000 draws of 23400 observations. Prices are simulated asynchronously and without noise. The abbreviation corresponds to the synchronization technique (HY = Hayashi-Yoshida, PT = previous tick interpolation, LI = Linear interpolation, and RT = Refresh Time) followed by the name of the estimator. Disentangled estimators are computed with 5 blocks.

\subsection{Synchronous prices \& noise}

We now study the impact of noise with synchronous trading. Our estimators are computed with sparse sampling (denoted by B at the end of the name; e.g. SpearB), sub-sampling (denoted by S), and preaveraging (denoted by P). We add the realized kernels of Barndorff-Nielsen et al. (2011) (denoted RK) to the comparison, and we skip results for 30 seconds, as it is well known to be too high frequency in the presence of noise.

Results are in Table 4 and three are the main conclusions. First, pre-averaging is the most efficient technique for dealing with microstructure noise, while sparse sampling gives the worse results. Sub- 
sampling -implemented on 5-minute returns- increases the efficiency of the estimates compared to sparse sampling, but provides higher RMSE than pre-averaged estimators. Yet, as the sampling frequency decreases from 1 to 5 minutes, DRC estimators based on blocks become more efficient, revealing the goodness of a sparser grid when prices are noisy. Results for 15 minutes-returns provide less clear cut results and advocates the use of 5 minute returns when using sparse sampling.

Second, for all approaches, the DRC estimator implemented with Gaussian ranks and Spearman's $\rho$ provide similar precision, except for model 1 for which Spearman's $\rho$ has smaller RMSE. These results, jointly with the milder condition for positive definiteness, supports the use of Gaussian ranks.

Third, when jumps are added the pre-averaged DRC estimators provide uniformly more precise estimates than the competitors. However, in absence of jumps realized kernels provide slightly more precise results. For 5-minute returns the two subsampled combinations perform better than most of the competitors except for OWC. Depending on the simulated model, one performs better than the other. The competing estimators are described by their respective authors as the least sensitive to noise when computed on basis of 5-minute returns. These statements are verified.

\subsection{Asynchronous prices \& noise}

Last, we analyze the goodness of the estimators with asynchronous and noisy observations. For the competing estimators, we focus on 5-minutes frequency As previous sub-sections showed that 5-minutes sampled observations gave the best results, we focus on this frequency. And since sparse sampling gave the worst results in previous subsections. The DRC estimators are shown using pre-averaging and subsampling. ${ }^{13}$

We synchronize using last tick interpolation at a frequency depending on the trading intensity of the considered securities. ${ }^{14}$ Note that Hayashi and Yoshida was used for the RC and TC estimators when data are clean of noise. However, if returns are noisy, HY becomes impracticable and hence the authors advise to use last tick interpolation. Realized kernels are implemented with refresh time and subsampled realized variances to compute to optimal bandwidth, following Barndorff-Nielsen et al. (2011).

Results are shown in Table 5. As in the case of asynchronous trading and noise, pre-averaging provides better estimates than subsampling. In the absence of jumps, realized kernels provide the best results, followed by the pre-averaged DRC. With jumps however the realized kernels provide inaccurate measures.

Pre-averaged DRC strike hence a good balance between jump-robustness and precision and form a serious alternative to other realized measures. Additionally, results are similar for DRC based on

\footnotetext{
${ }^{13}$ Pre-averaging is implemented on basis of the new grid of synchronous prices and subsampling is conducted with 5-minute returns also sampled from the new grid.

${ }^{14}$ We compute the $75 \%$ quantile of time intervals between trades for each asset, take the minimum and stamp the sampling frequency to the nearest second. The $75 \%$ quantile may be considered as a conservative choice but it allows to keep under control effects of asynchronous trading causing downward biases in correlations.
} 
Table 4: Monte Carlo study - Synchronous trading.

\begin{tabular}{|c|c|c|c|c|c|c|c|c|}
\hline & \multicolumn{2}{|c|}{ Model 1} & \multicolumn{2}{|c|}{ Model 2} & \multicolumn{2}{|c|}{ Model 3} & \multicolumn{2}{|c|}{ Model 4} \\
\hline & no jumps & jumps & no jumps & jumps & no jumps & jumps & no jumps & jumps \\
\hline \multicolumn{9}{|c|}{ Panel A: 1-minute returns } \\
\hline $\mathrm{RC}$ & 0.669 & 24.62 & 1.352 & 9.941 & 0.647 & 4.260 & 0.653 & 3.758 \\
\hline $\mathrm{BPC}$ & 0.717 & 2.978 & 1.369 & 2.474 & 0.683 & 1.095 & 0.691 & 1.178 \\
\hline TC & 0.648 & 0.668 & 1.314 & 1.347 & 0.626 & 0.654 & 0.632 & 0.655 \\
\hline OWC & 0.661 & 0.666 & 1.337 & 1.355 & 0.638 & 0.656 & 0.644 & 0.658 \\
\hline SpearB & 0.689 & 0.747 & 1.361 & 1.443 & 0.664 & 0.711 & 0.670 & 0.717 \\
\hline GaussB & 0.686 & 0.769 & 1.359 & 1.447 & 0.663 & 0.710 & 0.669 & 0.717 \\
\hline SpearS & 0.261 & 0.561 & 1.031 & 1.332 & 0.203 & 0.327 & 0.219 & 0.374 \\
\hline GaussS & 0.257 & 0.739 & 1.029 & 1.344 & 0.202 & 0.327 & 0.217 & 0.384 \\
\hline SpearP & 0.141 & 0.201 & 0.981 & 1.076 & 0.105 & 0.136 & 0.116 & 0.150 \\
\hline GaussP & 0.137 & 0.286 & 0.979 & 1.079 & 0.103 & 0.134 & 0.114 & 0.152 \\
\hline RK & 0.115 & 24.34 & 0.951 & 9.612 & 0.076 & 3.977 & 0.088 & 3.383 \\
\hline \multicolumn{9}{|c|}{ Panel B: 5-minute returns } \\
\hline $\mathrm{RC}$ & 0.323 & 24.42 & 1.033 & 9.659 & 0.224 & 3.931 & 0.251 & 3.462 \\
\hline $\mathrm{BPC}$ & 0.378 & 5.636 & 1.028 & 3.397 & 0.244 & 1.193 & 0.278 & 1.297 \\
\hline TC & 0.319 & 1.011 & 0.997 & 1.401 & 0.222 & 0.406 & 0.249 & 0.442 \\
\hline OWC & 0.329 & 0.368 & 1.009 & 1.141 & 0.223 & 0.297 & 0.252 & 0.325 \\
\hline SpearB & 0.411 & 0.709 & 1.106 & 1.425 & 0.315 & 0.439 & 0.343 & 0.487 \\
\hline GaussB & 0.404 & 0.764 & 1.100 & 1.423 & 0.314 & 0.437 & 0.340 & 0.485 \\
\hline SpearS & 0.261 & 0.561 & 1.031 & 1.332 & 0.203 & 0.327 & 0.219 & 0.374 \\
\hline GaussS & 0.257 & 0.739 & 1.029 & 1.344 & 0.202 & 0.327 & 0.217 & 0.384 \\
\hline SpearP & 0.141 & 0.201 & 0.981 & 1.076 & 0.105 & 0.136 & 0.116 & 0.150 \\
\hline GaussP & 0.137 & 0.286 & 0.979 & 1.079 & 0.103 & 0.134 & 0.114 & 0.152 \\
\hline RK & 0.115 & 24.34 & 0.951 & 9.612 & 0.076 & 3.977 & 0.088 & 3.383 \\
\hline \multicolumn{9}{|c|}{ Panel C: 15-minute returns } \\
\hline $\mathrm{RC}$ & 0.465 & 24.27 & 1.055 & 9.658 & 0.303 & 3.938 & 0.350 & 3.454 \\
\hline $\mathrm{BPC}$ & 0.553 & 9.850 & 1.036 & 5.070 & 0.341 & 1.844 & 0.400 & 1.891 \\
\hline $\mathrm{TC}$ & 0.468 & 6.195 & 1.021 & 3.758 & 0.319 & 1.406 & 0.363 & 1.401 \\
\hline OWC & 0.501 & 3.273 & 0.997 & 2.402 & 0.331 & 0.802 & 0.382 & 0.906 \\
\hline SpearB & 0.610 & 1.579 & 1.248 & 2.279 & 0.466 & 0.784 & 0.505 & 0.928 \\
\hline GaussB & 0.596 & 1.733 & 1.240 & 2.283 & 0.462 & 0.777 & 0.497 & 0.926 \\
\hline SpearS & 0.261 & 0.561 & 1.031 & 1.332 & 0.203 & 0.327 & 0.219 & 0.374 \\
\hline GaussS & 0.257 & 0.739 & 1.029 & 1.344 & 0.202 & 0.327 & 0.217 & 0.384 \\
\hline SpearP & 0.141 & 0.201 & 0.981 & 1.076 & 0.105 & 0.136 & 0.116 & 0.150 \\
\hline GaussP & 0.137 & 0.286 & 0.979 & 1.079 & 0.103 & 0.134 & 0.114 & 0.152 \\
\hline RK & 0.115 & 24.34 & 0.951 & 9.612 & 0.076 & 3.977 & 0.088 & 3.383 \\
\hline
\end{tabular}

Table 4 reports Monte Carlo simulation results for eight estimators of the integrated covariance matrix under four different models, specified with and without jumps. The entries report the root mean square errors (RMSE) computed as in (17) for 10000 draws of 23400 observations. Prices are simulated simultaneously and with noise. The abbreviations correspond to the the name of the estimator followed by the noise reduction technique $(\mathrm{B}=$ sparse sampling, $\mathrm{S}=$ sub-sampling, and $\mathrm{P}=$ pre-averaging). Disentangled estimators are computed with 5 blocks, except for 15-minute returns for which only 1 block is used. 
Table 5: Monte Carlo study - Asynchronous trading and noise.

\begin{tabular}{lcccccccc}
\hline & \multicolumn{2}{c}{ Model 1 } & \multicolumn{2}{c}{ Model 2 } & \multicolumn{2}{c}{ Model 3 } & \multicolumn{2}{c}{ Model 4 } \\
& no jumps & jumps & no jumps & jumps & no jumps & jumps & no jumps & jumps \\
\hline LT-RC & 0.404 & 17.47 & 4.625 & 9.318 & 0.386 & 2.965 & 0.264 & 2.439 \\
LT-BPC & 0.455 & 4.366 & 5.082 & 6.808 & 0.407 & 1.119 & 0.291 & 1.015 \\
LT-TC & 0.395 & 0.912 & 4.567 & 5.413 & 0.374 & 0.560 & 0.260 & 0.424 \\
LT-OWC & 0.404 & 0.455 & 4.550 & 4.897 & 0.376 & 0.477 & 0.264 & 0.331 \\
LT-SpearS & 0.344 & 0.650 & 4.599 & 5.226 & 0.355 & 0.495 & 0.233 & 0.365 \\
LT-GaussS & 0.341 & 0.820 & 4.598 & 5.255 & 0.355 & 0.496 & 0.231 & 0.374 \\
LT-SpearP & 0.184 & 0.295 & 1.366 & 1.527 & 0.145 & 0.191 & 0.144 & 0.191 \\
LT-GaussP & 0.180 & 0.454 & 1.365 & 1.550 & 0.143 & 0.191 & 0.141 & 0.199 \\
RK & 0.154 & 17.43 & 1.030 & 7.030 & 0.112 & 2.827 & 0.108 & 2.387 \\
\hline \hline
\end{tabular}

Table 5 reports Monte Carlo simulation results at 5-minute frequency and for six estimators of the integrated covariance matrix under four different models, specified with and without jumps. The entries report the root mean square errors (RMSE) computed as in (17) for 10000 draws of 23400 observations. Prices are simulated asynchronously and with noise. The abbreviation corresponds the used synchronization technique, followed by the name of the estimator and the noise reduction technique. Disentangled estimators are sub-sampled.

Spearman rho and Gaussian ranks. When jumps occur, they perform slightly less efficiently than OWC. Nevertheless, this drawback is compensated by a less demanding computational effort.

\section{Empirical application}

We now study the gains of our estimators from a financial perspective with a long-short portfolio management exercise. The investment universe is composed of 52 large stocks traded on the NYSE. Data consists of trades and prices. ${ }^{15}$ The data covers the period from October 2006 to April 2012 for a total of 1403 observations. The database is cleaned as in Barndorff-Nielsen et al. (2009).

Volatility timing strategies are based on conditional covariance matrix of daily returns, i.e. the covariance matrix at time $t+1$ given information up to time $t$. Since realized covariances are ex-post measures of the co-variation between assets, we use one-day ahead forecasts of the covariance matrix $\Sigma_{t}$ in the portfolio construction. The investor updates and rebalances the portfolio every day on basis of the new information generated by markets. If we consider a market composed of $p$ assets that the investor can select in his portfolio, the optimal $p \times 1$ vector of weights $\omega_{t}$ for the portfolio allocation are computed by solving standard conditional mean-variance criterion:

$$
\min _{\boldsymbol{\omega}_{t}}\left(\boldsymbol{\omega}_{t} \boldsymbol{\Sigma}_{t} \boldsymbol{\omega}_{t}\right)
$$

\footnotetext{
${ }^{15}$ The tickers are AA, ABT, AES, AKS, AMD, BMY, BSX, C, CAG, CBS, COH, CSX, CVX, D, DIS, DNR, EMC, EXC, FCX, GE, GIS, GLW, HAL, HPQ, HST, IRM, JCP, JPM, KEY, KO, MO, MRK, MS, NBR, NEM, ORCL, PFE, PG, RF, S, SLB, T, TJX, USB, VLO, VZ, WFC, WMT, WU, WY, XRX, SPY.
} 
subject to $\boldsymbol{\omega}_{t}^{\prime} \mathbf{1}=1$. The solution $\boldsymbol{\omega}_{t}$ to this strategy is well known:

$$
\boldsymbol{\omega}_{t}=\frac{\boldsymbol{\Sigma}_{t}^{-1} \mathbf{1}}{\mathbf{1}^{\prime} \Sigma_{t}^{-1} \mathbf{1}}
$$

Minimum variance portfolios can produce large negative and highly volatile weights. Adding $L_{1}$ constraints to the minimization problem in known to produce more realistic results as underlined by Fan et al. (2009). Therefore, we also test the previous problem with an additional constraint $\left|\boldsymbol{\omega}_{t}\right|^{\prime} \mathbf{1} \leq 1+2 m$ where $m$ provides the short positions boundary. Following Boudt et al. (2012), we set $m=20 \%$ which allows to construct $120 / 20$ portfolios. Solutions to this problem are provided from quadratic programing solvers.

We evaluate the performance of the portfolio on basis of five criteria: i) the annualized mean returns, ii) the annualized standard deviation, iii) the annualized Sharp ratio, iv) the annualized average turnover (given by $\mathrm{TO}_{t}=\left|\boldsymbol{\omega}_{t}-\boldsymbol{\omega}_{t-1}\right| \mathbf{1}_{p}$, where $\mathbf{1}_{p}$ is a vector of ones), and $\mathrm{v}$ ) the cumulated performance in terms of the return on investment.

\subsection{Models for conditional covariance matrices}

We consider a set of $p$ stocks and denote the daily returns at $t$ by $\mathbf{r}_{t}$ and realized covariance measures on day $t$ as $\mathbf{V}_{t}$. Investors believe that asset returns behave as:

$$
\mathbf{r}_{t}=\boldsymbol{\mu}+\Sigma_{t}^{\frac{1}{2}} \mathbf{z}_{t}
$$

where $\mathbf{z}_{t} \sim$ i.i.d. $N\left(0, \mathbf{I}_{p}\right)$ and $\mathbf{I}_{p}$ denotes the identity matrix of size $p, \Sigma_{t}=V\left[\mathbf{r}_{t} \mid \mathcal{A}_{t-1}\right]$ and $\mathcal{A}_{t-1}$ is the information set.

Three models are considered for $\Sigma_{t}$. Two of them rely on realized covariances and one on daily returns only. In other words, we compare investments conducted on basis of two different information sets: $\mathcal{A}_{t}^{L F}$ and $\mathcal{A}_{t}^{H F}$. The low frequency information set $\mathcal{A}_{t}^{L F}$ is generated by daily returns, and the high frequency information set $\mathcal{A}_{t}^{H F}$ is generated by daily returns and realized covariances. More rigorously we have: $\mathcal{A}_{t}^{L F}=\sigma\left(\left\{\mathbf{r}_{s}\right\}, s \leq t\right)$ and $\mathcal{A}_{t}^{H F}=\sigma\left(\left\{\mathbf{r}_{s}, \mathbf{V}_{s}\right\}, s \leq t\right)$.

The low-frequency benchmark model is the DCC of Engle (2002):

$$
\begin{aligned}
& \boldsymbol{\Sigma}_{t}=\mathbf{D}_{t} \mathbf{R}_{t} \mathbf{D}_{t} \text { where } \\
& \mathbf{R}_{t}=\left(\mathbf{Q}_{t} \odot \mathbf{1}_{p}\right)^{-1 / 2} \mathbf{Q}_{t}\left(\mathbf{Q}_{t} \odot \mathbf{1}_{p}\right)^{-1 / 2}, \\
& \mathbf{Q}_{t}=(1-\alpha-\beta) \overline{\mathbf{Q}}+\alpha \mathbf{u}_{t-1} \mathbf{u}_{t-1}^{\prime}+\beta \mathbf{Q}_{t-1},
\end{aligned}
$$

$\mathbf{D}_{t}=\left(\boldsymbol{\Sigma}_{t} \odot \mathbf{I}_{p}\right)^{1 / 2}, u_{i, t}=z_{i, t} / h_{i, t}^{1 / 2}$, and $h_{i, t}^{1 / 2}$ denotes the elements on the diagonal of $\mathbf{D}_{t}$, i.e. the univariate volatilities which are specified as $\mathrm{GARCH}(1,1)$ processes. Long memory is not considered since only one step ahead forecasts are required.

We consider two models for the conditional covariance matrix based on high-frequency data. First, 
we estimate a rolling window volatility model. ${ }^{16}$ The estimation procedure closely follows De Pooter et al. (2008) and Fleming et al. (2003). This technique is based on the work of Foster \& Nelson (1996) and Andreou \& Ghysels (2002). The daily conditional covariance matrix based on high-frequency data is:

$$
\Sigma_{t}=\exp (-\alpha) \boldsymbol{\Sigma}_{t-1}+\alpha \exp (-\alpha) \mathbf{V}_{t-1}
$$

Smaller values for the decay parameter $\alpha$ point to a less informative innovation process $\mathbf{V}_{t-1}$, i.e. the estimator is too noisy from a portfolio construction perspective, while a large value for $\alpha$ points to more informative innovations (Bannouh et al. (2009)). Fleming et al. (2003) and De Pooter et al. (2008) point out that statistically optimal parameters do not lead to optimal financial performances. Indeed, estimating $\alpha$ via maximum likelihood does not provide the portfolio with the best risk-return trade-off. This is why Boudt et al. (2012) consider two optimality criteria: maximum likelihood and minimum volatility of the investment. Yet, to be fair with the other two models, we use maximum likelihood.

The second model with high frequency data is the HEAVY of Noureldin et al. (2012). Their model is specified as the BEKK of Engle \& Kroner (1995) but lagged values of the cross products of returns are replaced by lagged values of the realized covariances:

$$
\Sigma_{t}=\mathbf{\Omega}+\mathbf{B} \boldsymbol{\Sigma}_{t-1} \mathbf{B}^{\prime}+\mathbf{A V}_{t-1} \mathbf{A}^{\prime}
$$

Realized covariances are modeled as $E\left[\mathbf{V}_{t} \mid \mathcal{A}_{t-1}^{H F}\right]=\mathbf{M}_{t}$ that is again specified as a BEKK:

$$
\mathbf{M}_{t}=\mathbf{\Omega}_{M}+\mathbf{D M}_{t-1} \mathbf{D}^{\prime}+\mathbf{G V}_{t-1} \mathbf{G}^{\prime} .
$$

Various specifications can be adopted to limit the amount of parameters. We study the scalar-BEKK specification whose properties are illustrated in the empirical study of Noureldin et al. (2012). The Wishart distribution is assumed and estimation is done by maximum likelihood.

\subsection{Results}

Results of the unconstrained minimum variance portfolio are in Table 6 and Figure 3. Results for the constrained version are very similar, suggesting that the constraints are not reached frequently. More details are available in Table 8 of the Appendix. Table 6 summarizes the performance for portfolios constructed on basis of the rolling window model, the HEAVY model and the DCC. Results for the DCC are reported under the results of the HEAVY model (at the bottom right corner). Mean returns (denoted Mean) and standard deviations (denoted Std. dev.) are annualized, while turnover (denoted TO) is daily. The fifth performance measure is displayed in the figure, which shows the cumulated Return On Investment of one monetary unit invested in October 2006 for the different estimators. All the performances based on realized measures are located in the shaded area (delimited by the minimum and the maximum daily performances). Other lines illustrate performances on the same period for the strategy

\footnotetext{
16 "Rolling window" is the name of the model, which is different to rolling unconditional estimation by moving a window of observations.
} 
Table 6: Unconstrained minimum variance portfolio - Performance measures.

\begin{tabular}{lccccccccc}
\hline \hline & \multicolumn{3}{c}{ Rolling Window } \\
Model & Mean & Sdt dev. & Sharp & TO & $\alpha$ & Mean & Sdt dev. & Sharp & TO \\
\hline RC & $9.76 \%$ & $12.51 \%$ & 0.780 & 0.15 & 0.048 & $12.61 \%$ & $12.25 \%$ & 1.029 & 0.97 \\
BPC & $8.27 \%$ & $12.90 \%$ & 0.641 & 0.14 & 0.043 & $13.45 \%$ & $12.57 \%$ & 1.071 & 0.84 \\
TC & $8.35 \%$ & $12.84 \%$ & 0.651 & 0.10 & 0.038 & $12.50 \%$ & $12.30 \%$ & 1.016 & 1.02 \\
OWC & $9.69 \%$ & $12.94 \%$ & 0.749 & 0.11 & 0.036 & $12.24 \%$ & $12.31 \%$ & 0.995 & 0.30 \\
RK & $8.42 \%$ & $12.76 \%$ & 0.659 & 0.14 & 0.046 & $10.84 \%$ & $12.53 \%$ & 0.865 & 0.96 \\
SpearS & $7.00 \%$ & $13.41 \%$ & 0.522 & 0.08 & 0.029 & $13.57 \%$ & $12.42 \%$ & 1.093 & 0.49 \\
GaussS & $6.47 \%$ & $13.39 \%$ & 0.483 & 0.08 & 0.029 & $13.87 \%$ & $12.44 \%$ & 1.115 & 0.51 \\
SpearP & $7.63 \%$ & $13.19 \%$ & 0.579 & 0.10 & 0.036 & $11.64 \%$ & $12.46 \%$ & 0.934 & 0.20 \\
GaussP & $7.20 \%$ & $13.16 \%$ & 0.547 & 0.10 & 0.038 & $11.76 \%$ & $12.43 \%$ & 0.947 & 1.09 \\
\hline DCC & - & - & - & - & - & $6.52 \%$ & $13.39 \%$ & 0.487 & 0.54 \\
\hline \hline
\end{tabular}

Table 6 summarizes the performance for portfolios constructed on basis of the rolling window model, the HEAVY model and the DCC. Results for the DCC are reported under the results of the HEAVY model. Mean returns and standard deviations are annualized while turnover is on daily basis.

based on the DDC (solid line), and the Spider S\&P500 ETF (SPY; dashed). The solid black line, denoted DRC-GR, represents the performance of the portfolio based on the disentangled realized covariances with Gaussian ranks.

The strategy based on the HEAVY model is more profitable (mean returns) than based on the rolling window model. It is also less risky (of the order of 12.4\%). Compared with the average S\&P500 VIX over the sample period (24.66\%), the volatility of the portfolio using the HEAVY model is quite low. Moreover, on average, the Sharp ratios are higher than for the rolling window model. These results are not surprising since the HEAVY model has a richer specification than the rolling window. Nevertheless, the portfolios constructed on basis of the HEAVY model are less stable as the turnover is higher. The $D C C$ behaves worse that the other models. The average return is lower while the annualized volatility remains similar to other strategies. This performance may be due to the fact that the DCC is based on a weaker information set.

The return on investment (see figure) confirms that investing on basis of the HEAVY model is preferable to the rolling window and the $D C C$, while investing in the market portfolio through the S\&P 500 ETF did not generate any gain. The return using our preferred estimator and HEAVY reaches 100\%, which beats the alternatives.

\section{Conclusion}

We study the class of disentangled estimators of the integrated covariance matrix of Itō semimartingales with an extensive Monte Carlo study. We cover different scenarios when efficient prices are observable or contaminated by noise, with and without jumps, and with synchronous or non-synchronous trading. We show that if one selects the right combinations of estimators and robustification techniques, disentangled 


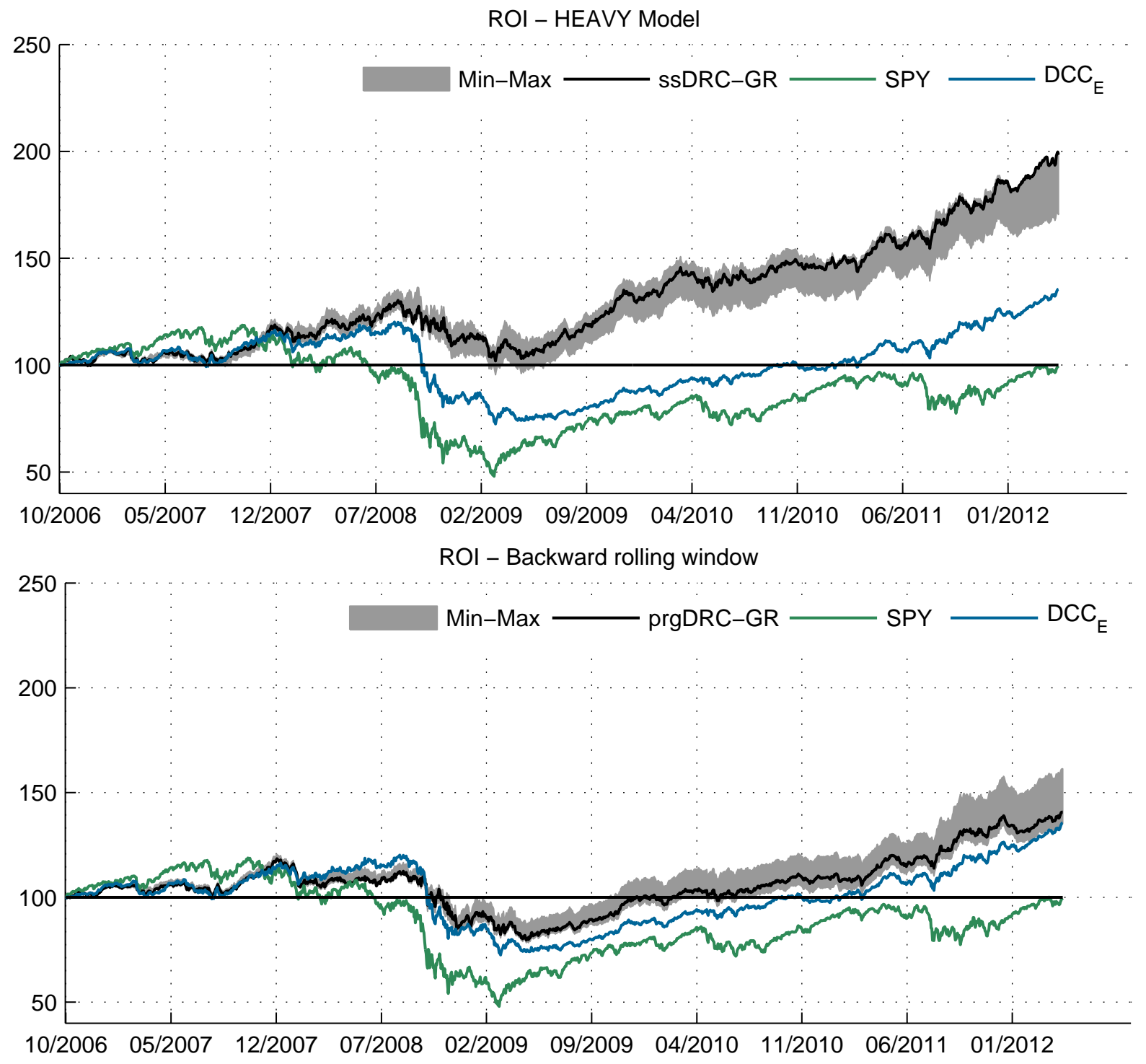

Figure 3: Cumulated performance for the different estimators in terms of return on investment of 100 monetary unit invested in October 2006. All the performances are located in the shaded area delimited by the minimum and the maximum daily performance. Other lines illustrate performances on the same period for the strategy based on the DCC and the performance of a S\&P500 ETF. The solid black line represents the performance of the portfolio based on the Gaussian ranks disentangled realized covariances with subsampling and pre-averaging.

realized covariances prove to be precise, jump robust, simple, robust to noise, positive definite, and computationally efficient. Our main conclusion is that if observations are non-synchronous and noisy (as it is the case of multivariate high frequency data), the pre-averaged version of disentangled estimators based on Gaussian ranks (for the correlations) and median deviations (for the volatilities) is the most appropriate metrics in terms of root mean square error. This result dovetails with Boudt et al. (2012). Moreover, this finding is confirmed by an empirical analysis based on a cross-section of stocks traded on 
the NYSE. The best estimator in the Monte Carlo study also performs the best on a minimum variance portfolio strategy. It provides the highest mean return, lower volatility, highest Sharp ratio, and highest return on investment. 


\section{Appendix: supplementary Monte Carlo results}

Table 7: Monte Carlo study - Synchronous trading.

\begin{tabular}{|c|c|c|c|c|c|c|c|c|}
\hline & \multicolumn{2}{|c|}{ Model 1} & \multicolumn{2}{|c|}{ Model 2} & \multicolumn{2}{|c|}{ Model 3} & \multicolumn{2}{|c|}{ Model 4} \\
\hline & no jumps & jumps & no jumps & jumps & no jumps & jumps & no jumps & jumps \\
\hline \multicolumn{9}{|c|}{ Panel A: 30-seconds returns } \\
\hline IQR-Ken & 0.109 & 0.117 & 0.981 & 1.038 & 0.087 & 0.092 & 0.094 & 0.099 \\
\hline IQR-Spear & 0.109 & 0.116 & 0.982 & 1.039 & 0.087 & 0.092 & 0.094 & 0.100 \\
\hline IQR-Quad & 0.135 & 0.140 & 0.982 & 1.039 & 0.090 & 0.095 & 0.104 & 0.107 \\
\hline IQR-Gauss & 0.106 & 0.136 & 0.980 & 1.038 & 0.086 & 0.090 & 0.091 & 0.098 \\
\hline \multicolumn{9}{|c|}{ Panel B: 1-minute returns } \\
\hline IQR-Ken & 0.159 & 0.181 & 1.028 & 1.114 & 0.127 & 0.144 & 0.136 & 0.153 \\
\hline IQR-Spear & 0.160 & 0.179 & 1.030 & 1.118 & 0.127 & 0.145 & 0.137 & 0.156 \\
\hline IQR-Quad & 0.194 & 0.208 & 1.031 & 1.116 & 0.131 & 0.148 & 0.149 & 0.166 \\
\hline IQR-Gauss & 0.154 & 0.211 & 1.025 & 1.114 & 0.125 & 0.141 & 0.133 & 0.152 \\
\hline \multicolumn{9}{|c|}{ Panel C: 5-minute returns } \\
\hline IQR-Ken & 0.451 & 1.394 & 1.382 & 1.936 & 0.367 & 0.585 & 0.387 & 0.641 \\
\hline IQR-Spear & 0.464 & 1.396 & 1.392 & 1.962 & 0.374 & 0.597 & 0.396 & 0.660 \\
\hline IQR-Quad & 0.522 & 1.389 & 1.391 & 1.941 & 0.373 & 0.590 & 0.411 & 0.650 \\
\hline IQR-Gauss & 0.440 & 1.426 & 1.374 & 1.930 & 0.362 & 0.578 & 0.381 & 0.634 \\
\hline \multicolumn{9}{|c|}{ Panel D: 15-minute returns } \\
\hline IQR-Ken & 0.648 & 2.518 & 1.418 & 3.022 & 0.501 & 0.961 & 0.534 & 1.152 \\
\hline IQR-Spear & 0.659 & 2.540 & 1.424 & 3.066 & 0.503 & 0.974 & 0.539 & 1.177 \\
\hline IQR-Quad & 0.771 & 2.395 & 1.455 & 3.038 & 0.518 & 0.986 & 0.583 & 1.193 \\
\hline IQR-Gauss & 0.634 & 2.653 & 1.406 & 3.024 & 0.495 & 0.952 & 0.523 & 1.148 \\
\hline
\end{tabular}

Table 7 reports Monte Carlo estimation results for 4 quantile-based volatility estimators of the integrated covariance matrix under four different models, specified with and without jumps. The columns report the root mean square errors (RMSE) as computed in (17) for 4000 draws of 23400 observations each corresponding to a situation of one day of 6.5 hours of trading and prices recorded simultaneously each seconds. 
Table 8: Constrained minimum variance portfolio - Performance measures.

\begin{tabular}{|c|c|c|c|c|c|c|c|c|c|}
\hline \multirow[b]{2}{*}{ Model } & \multicolumn{5}{|c|}{ Rolling Window } & \multicolumn{4}{|c|}{ HEAVY } \\
\hline & Mean & Sdt dev. & Sharp & $\mathrm{TO}$ & $\alpha$ & Mean & Sdt dev. & Sharp & $\mathrm{TO}$ \\
\hline $\mathrm{RC}$ & $9.76 \%$ & $12.51 \%$ & 0.780 & 0.15 & 0.048 & $12.60 \%$ & $12.25 \%$ & 1.029 & 0.97 \\
\hline $\mathrm{BPC}$ & $8.27 \%$ & $12.90 \%$ & 0.641 & 0.14 & 0.043 & $13.43 \%$ & $12.57 \%$ & 1.068 & 0.84 \\
\hline TC & $8.35 \%$ & $12.84 \%$ & 0.651 & 0.10 & 0.038 & $12.55 \%$ & $12.30 \%$ & 1.020 & 1.02 \\
\hline OWC & $9.69 \%$ & $12.94 \%$ & 0.749 & 0.11 & 0.036 & $12.22 \%$ & $12.30 \%$ & 0.993 & 0.30 \\
\hline RK & $8.42 \%$ & $12.76 \%$ & 0.659 & 0.14 & 0.046 & $10.83 \%$ & $12.53 \%$ & 0.864 & 0.96 \\
\hline SpearS & $7.00 \%$ & $13.41 \%$ & 0.522 & 0.08 & 0.029 & $13.40 \%$ & $12.41 \%$ & 1.080 & 0.47 \\
\hline GaussS & $6.46 \%$ & $13.39 \%$ & 0.483 & 0.08 & 0.029 & $13.33 \%$ & $12.40 \%$ & 1.075 & 0.46 \\
\hline SpearP & $7.63 \%$ & $13.19 \%$ & 0.579 & 0.10 & 0.036 & $11.64 \%$ & $12.47 \%$ & 0.934 & 0.20 \\
\hline GaussP & $7.20 \%$ & $13.16 \%$ & 0.547 & 0.10 & 0.038 & $11.66 \%$ & $12.41 \%$ & 0.940 & 1.09 \\
\hline$D C C$ & - & - & - & - & - & $6.69 \%$ & $13.31 \%$ & 0.503 & 0.53 \\
\hline
\end{tabular}

Table 8 reports the performance for portfolios constructed on basis of the rolling window model, the HEAVY model and the DCC. Results for the DCC are reported under the results of the HEAVY model. Mean returns and standard deviations have been annualized while turnover is on daily basis.

\section{References}

Aït-Sahalia, Y., Fan, J., \& Xiu, D. (2010). High-frequency covariance estimates with noisy and asynchronous data. Journal of the American Statistical Association, 105, 1504-1517.

Andersen, T. G. \& Bollerslev, T. (1998). Answering the skeptics: Yes, standard volatility models do provide accurate forecasts. International Economic Review, 39, 885905.

Andersen, T. G., Bollerslev, T., Diebold, F., \& Labys, P. (2001). The distribution of realized exchange rate volatility. Journal of the American statistical association, 96, 42-55.

Andersen, T. G., Bollerslev, T., Diebold, F., \& Labys, P. (2003). Modeling and forecasting realized volatility. Econometrica, 71(2), 579-625.

Andersen, T. G., Dobrev, D., \& Schaumburg, E. (2012). Jump-robust volatility estimation using nearest neighbor truncation. Journal of Econometrics, 169(1), 75-93.

Andreou, E. \& Ghysels, E. (2002). Rolling-sample volatility estimators. Journal of Business E Economic Statistics, 20(3).

Bandi, F. M. \& Russell, J. R. (2008). Microstructure noise, realized variance, and optimal sampling. The Review of Economic Studies, 75(2), 339-369.

Bannouh, K., van Dijk, D., \& Martens, M. (2009). Range-based covariance estimation using high-frequency data: the realized co-range. Journal of Financial Econometrics, 7, 341372.

Barndorff-Nielsen, O. E., Hansen, P. R., Lunde, A., \& Shephard, N. (2008). Designing realized kernels to measure the ex post variation of equity prices in the presence of noise. Econometrica, 76(6), 1481-1536. 
Barndorff-Nielsen, O. E., Hansen, P. R., Lunde, A., \& Shephard, N. (2009). Realized kernels in practice: Trades and quotes. The Econometrics Journal, 12(3), C1-C32.

Barndorff-Nielsen, O. E., Hansen, P. R., Lunde, A., \& Shephard, N. (2011). Multivariate realised kernels: Consistent positive semi-definite estimators of the covariation of equity prices with noise and nonsynchronous trading. Journal of Econometrics, 162(2), 149-169.

Barndorff-Nielsen, O. E. \& Shephard, N. (2004a). Measuring the impact of jumps in multivariate price processes using bipower covariation. Discussion Paper, Nuffield College, Oxford University.

Barndorff-Nielsen, O. E. \& Shephard, N. (2004b). Power and bipower variation with stochastic volatility and jumps. Journal of financial econometrics, 2(1), 1-37.

Barndorff-Nielsen, O. E. \& Shephard, N. (2007). Variation, jumps, market frictions and high frequency data in financial econometrics. In Advances in Economics and Econometrics. Theory and Applications, Ninth World Congress. vol. 3, 328-372.

Bauwens, L., Laurent, S., \& Rombouts, J. V. (2006). Multivariate garch models: a survey. Journal of Applied Econometrics, 21(1), 79-109.

Bollerslev, T. (1990). Modelling the coherence in short-run nominal exchange rates: a multivariate generalized arch model. The Review of Economics and Statistics, 72, 498-505.

Boudt, K., Cornelissen, J., \& Croux, C. (2011a). The gaussian rank correlation estimator: robustness properties. Statistics and Computing, 22, 471-483.

Boudt, K., Cornelissen, J., \& Croux, C. (2012). Jump robust daily covariance estimation by disentangling variance and correlation components. Computational Statistics and Data Analysis, 56(11), 2993-3005.

Boudt, K., Croux, C., \& Laurent, S. (2011b). Outlyingness weighted covariation. Journal of Financial Econometrics, 9(4), 657-684.

Boudt, K. \& Zhang, J. (2013). Jump robust two time scale covariance estimation and realized volatility budgets. Quantitative Finance, (ahead-of-print), 1-14.

Carr, P., Geman, H., Madan, D. B., \& Yor, M. (2002). The fine structure of asset returns: An empirical investigation. Journal of Business, 75(2), 305-332.

Christensen, K., Kinnebrock, S., \& Podolskij, M. (2010a). Pre-averaging estimators of the ex-post covariance matrix in noisy diffusion models with non-synchronous data. Journal of Econometrics, 159(1), 116-133.

Christensen, K., Oomen, R., \& Podolskij, M. (2010b). Realised quantile-based estimation of the integrated variance. Journal of Econometrics, 159(1), 74-98. 
Christensen, K., Podolskij, M., \& Vetter, M. (2013). On covariation estimation for multivariate continuous itô semimartingales with noise in non-synchronous observation schemes. Journal of Multivariate Analysis, $120,59-84$.

Corsi, F. \& Audrino, F. (2012). Realized covariance tick-by-tick in presence of rounded time stamps and general microstructure effects. Journal of Financial Econometrics, nbs007.

Corsi, F., Peluso, S., \& Audrino, F. (2014). Missing in asynchronicity: A kalman-em approach for multivariate realized covariance estimation. Journal of Applied Econometrics.

Dacorogna, M., Gencay, R., Muller, U., Olsen, R., \& Pictet, O. (2001). An Introduction to High Frequency Finance. Academic Press.

De Pooter, M., Martens, M., \& Van Dijk, D. (2008). Predicting the daily covariance matrix for S\&P 100 stocks using intraday data but which frequency to use? Econometric Reviews, 27, 199229.

Engle, R. (2002). Dynamic conditional correlation: A simple class of multivariate generalized autoregressive conditional heteroskedasticity models. Journal of Business \& Economic Statistics, 20(3), 339-350.

Engle, R. \& Kroner, K. (1995). Multivariate simultaneous generalized arch. Econometric theory, 11(01), $122-150$.

Epps, T. W. (1979). Comovements in stock prices in the very short run. Journal of the American Statistical Association, 74(366a), 291-298.

Fan, J., Li, Y., \& Yu, K. (2012). Vast volatility matrix estimation using high-frequency data for portfolio selection. Journal of the American Statistical Association, 107(497), 412-428.

Fan, J., Zhang, J., \& Yu, K. (2009). Asset allocation and risk assessment with gross exposure constraints for vast portfolios. Technical report, Princeton University.

Fleming, J., Kirby, C., \& Ostdiek, B. (2003). The economic value of volatility timing using realized volatility. Journal of Financial Economics, 67(3), 473-509.

Foster, D. P. \& Nelson, D. B. (1996). Continuous record asymptotics for rolling sample variance estimators. Econometrica, 64(3), 139174.

Halbleib, R. \& Voev, V. (2011). Forecasting Covariance Matrices: A Mixed Frequency Approach. CREATES Research Papers - 2011-03.

Harris, F., McInish, T., Shoesmith, G., \& Wood, R. (1995). Cointegration, error correction and price discovery on informationally-linked security markets. Journal of Financial and Quantitative Analysis, 30, 563-581.

Hautsch, N., Kyj, L. M., \& Oomen, R. (2012). A blocking and regularization approach to high-dimensional realized covariance estimation. Journal of Applied Econometrics, 27(4), 625-645. 
Hayashi, T. \& Yoshida, N. (2005). On covariance estimation of non-synchronously observed diffusion processes. Bernoulli, 11(2), 359-379.

Jacod, J., Li, Y., Mykland, P. A., Podolskij, M., \& Vetter, M. (2009). Microstructure noise in the continuous case: the pre-averaging approach. Stochastic Processes and their Applications, 119(7), 2249-2276.

Jacod, J. \& Todorov, V. (2009). Testing for common arrivals of jumps for discretely observed multidimensional processes. The Annals of Statistics, 1792-1838.

Mancini, C. \& Gobbi, F. (2012). Identifying the brownian covariation from the co-jumps given discrete observations. Econometric Theory, 28(2), 249-273.

Martens, M. (2004). Estimating unbiased and precise realized covariances. Department of Finance, Erasmus School of Economics, Rotterdam.

McAleer, M. \& Medeiros, M. C. (2008). Realized volatility: A review. Econometric Reviews, 27(1-3), 10-45.

Mykland, P. A. \& Zhang, L. (2009). Inference for continuous semimartingales observed at high frequency. Econometrica, 77(5), 1403-1445.

Noureldin, D., Shephard, N., \& Sheppard, K. (2012). Multivariate high-frequency-based volatility (heavy) models. Journal of Applied Econometrics, 27(6), 907-933.

Podolskij, M. \& Vetter, M. (2009). Estimation of volatility functionals in the simultaneous presence of microstructure noise and jumps. Bernoulli, 15(3), 634-658.

Protter, P. E. (2004). Stochastic Integration and Differential Equations: Version 2.1, vol. 21. Springer.

Reno, R. (2003). A closer look at the epps effect. International Journal of Theoretical and Applied Finance, 6(01), 87-102.

Rousseeuw, P. J. \& Croux, C. (1993). Alternatives to the median absolute deviation. Journal of the American Statistical Association, 88, 1273-1283.

Shephard, N. \& Xiu, D. (2012). Econometric analysis of multivariate realised qml: estimation of the covariation of equity prices under asynchronous trading. Tech. rep., University of Oxford and University of Chicago.

Shevlyakov, G. \& Smirnov, P. (2011). Robust estimation of the correlation coefcient: An attempt of survey. Austrian Journal of Statistics, 40(1E2), 147-156.

Tse, Y. K. \& Tsui, A. (2000). A multivariate garch model with time-varying correlations. Available at SSRN $250228,20,351-362$.

Voev, V. \& Lunde, A. (2007). Integrated covariance estimation using high-frequency data in the presence of noise. Journal of Financial Econometrics, 5(1), 68-104. 
Zhang, L. (2006). Efficient estimation of stochastic volatility using noisy observations: A multi-scale approach. Bernoulli, 12(6), 1019-1043.

Zhang, L. (2011). Estimating covariation: Epps effect, microstructure noise. Journal of Econometrics, 160, 33-47.

Zhang, L., Mykland, P., \& Aït-Sahalia, Y. (2005). A tale of two time scales: Determining integrated volatility with noisy high frequency data. Journal of The American Statistical Association, 100(472), 1394-1411.

Zhou, B. (1996). High-frequency data and volatility in foreign-exchange rates. Journal of Business $\mathcal{E}$ Economic Statistics, 14(1), 45-52. 